\title{
Optimal requirements of a data acquisition system for a quadrupolar probe used in resistivity and permittivity surveys
}

\author{
Alessandro Settimi ${ }^{1{ }^{\star}}$, Achille Zirizzotti ${ }^{1}$, James A. Baskaradas ${ }^{1}$, Cesidio Bianchi ${ }^{1}$ \\ ${ }^{1}$ Istituto Nazionale di Geofisica e Vulcanologia, Sezione Roma 2, Roma, Italy
}

\section{Article history}

Received May 27, 2010; accepted July 23, 2010.

Subject classification:

Instrumentation and techniques of general interest, Magnetic and electrical methods, Mathematical geophysics, Data processing.

\section{ABSTRACT}

This report discusses the development and engineering of a suitable quadrupolar probe for simultaneous and noninvasive surveys of electrical resistivity and dielectric permittivity. The quadrupolar probe can perform measurements on a subsurface with inaccuracies below a fixed limit (10\%) in a bandwidth of low frequency $(100 \mathrm{kHz})$. The quadrupole should be connected to an appropriate analog-digital converter that samples in phase and quadrature (IQ) or in uniform mode. If the probe is characterized by a galvanic contact with the surface, the inaccuracies in the measurements of resistivity and permittivity due to the IQ or uniform sampling analogdigital converter are analytically expressed. A large number of numerical simulations show that the performances of the probe depend on the selected sampler, and that the IQ is better compared to the uniform mode under the same operating conditions, i.e. for bit resolution and medium.

\section{Introduction}

\section{Analog-digital converter}

Typically, an analog-digital converter (ADC) [Razavi 1995] is an electronic device that converts an input analog voltage (or current) into a digital number. A sampler has several sources of errors. The accuracy is mainly limited by quantitation error. However, accurate reproduction is only possible if the sampling rate is more than twice the highest frequency of the signal. This is essentially what is embodied in the Shannon-Nyquist sampling theorem.

Different users of sampler models are interested in different modeling details, and so numerous models have been proposed in the literature. Some of these describe specific error sources [Polge et al. 1975], others are devised to connect conversion techniques and the corresponding errors [Arpaia et al. 1999, Arpaia et al. 2004].

\section{Resistivity and permittivity surveys}

Electrical resistivity and dielectric permittivity are two independent physical properties that characterize the behavior of bodies excited by an electromagnetic field. The measurements of these properties provides crucial information regarding the practical uses of bodies (for example, materials that conduct electricity), and for countless other purposes.

Some studies have shown that the electrical resistivity and dielectric permittivity of a body can be obtained by measuring the complex impedance using a system with four electrodes, although these electrodes do not require resistive contact with the investigated body [Grard 1990a, Grard 1990b, Grard and Tabbagh 1991, Tabbagh et al. 1993, Vannaroni et al. 2004, Del Vento and Vannaroni 2005]. In this case, the current is made to circulate in the body by electric coupling, supplying the electrodes with an alternating electrical signal of low (LF) or middle (MF) frequency. In this type of investigation, the range of the optimal frequencies for electrical resistivity values of the more common materials is between $\approx 10 \mathrm{kHz}$ and $\approx 1 \mathrm{MHz}$. Once complex impedance has been acquired, the distributions of the resistivity and permittivity in the investigated body can be estimated using well-known algorithms of inversion techniques.

Applying the same principle, but limited to the acquisition of resistivity only, there are various commercial instruments that are used in geology for investigating the first 2-5 $\mathrm{m}$ beneath the ground, both for the exploration of environmental areas and for archeological investigations [Samouëlian et al. 2005].

For the direct determination of the dielectric permittivity in the subsoil, besides geo-radar that can provide an estimate by complex measurement procedures on radar-gram processing [Declerk 1995, Sbartaï et al. 2006], the only technical instrument currently in use is known as the time-domain reflectometer. Time-domain reflectometers use two electrodes that are inserted deep into the ground, to acquire the dielectric permittivity in the subsoil for further analysis [Mojid et al. 2003, Mojid and Cho 2004].

\section{Topic and structure of the report}

This report presents a discussion of theoretical modeling, and moves towards the practical implementation of a quadrupolar probe that acquires complex impedance in 


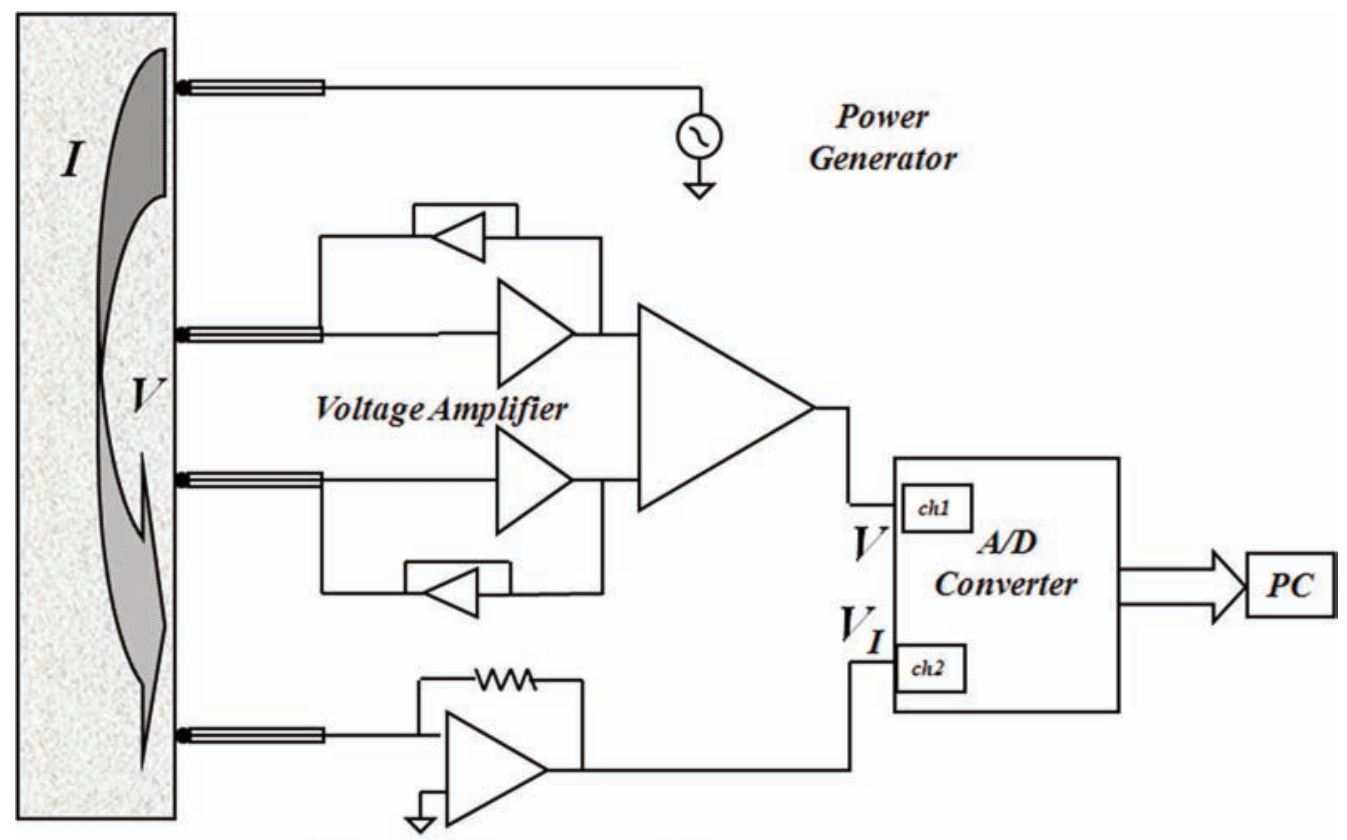

Trans-Resistance Amplifier

Figure 1. Block diagram of the measuring system that is composed of: a series of four electrodes laid on the material to be investigated; an analog circuit for the detection of signals, connected to a high voltage sinusoidal generator; a digital acquisition system; and a personal computer. The four electrodes are laid on the block of material to be analyzed. Two electrodes are used to generate and measure the injected current (at a selected frequency), while the other two electrodes are used to measure the potential difference. In this way, two voltages are obtained: the first proportional to the current; the second proportional to the difference of potential. These voltages are digitized through a analog-digital converter (A/D converter) connected to a personal computer (PC) for further processing. The real magnitudes hereby measured in the time domain are subsequently transformed into complex magnitudes in the frequency domain. From the ratio of the complex values, at the specific investigated frequency, it is possible to obtain the complex impedance. A program with an algorithm of numerical inversion allows the electrical resistivity and dielectric permittivity of the material to be obtained by measuring the transfer impedance; in this way, the reliability of the measured data is immediately analyzed, which is very useful during a measurement program.

the field. Quadrupolar probes allow the measurement of electrical resistivity and dielectric permittivity using alternating current at $\mathrm{LF}(30 \mathrm{kHz}<\mathrm{f}<300 \mathrm{kHz})$ or MF $(300 \mathrm{kHz}<\mathrm{f}<3 \mathrm{MHz})$. By increasing the distance between the electrodes, it is possible to investigate the electrical properties of sub-surface structures at greater depths. With the appropriate arrangements, measurements can be carried out with the electrodes slightly raised above the surface, providing completely nondestructive analysis, although also associated with greater errors. The probe can be used to perform immediate measurements on materials with high resistivity and permittivity, without the subsequent stages of data analysis.

In our recent study [Settimi et al. 2010], we proposed the theoretical modeling of the simultaneous and noninvasive measurement of electrical resistivity and dielectric permittivity using a quadrupole probe on a subjacent medium. A mathematical-physical model was applied on the propagation of errors in the measurement of resistivity and permittivity, based on the sensitivity functions tool. The findings were also compared with the results of the classical method of analysis in the frequency domain, which is useful for determining the behavior of zero and pole frequencies in the linear time-invariant circuit of the quadrupole. We underlined that the average values of electrical resistivity and dielectric permittivity can be used to estimate the complex impedance over various terrains and concretes, especially when they are characterized by low levels of water saturation or content, and are analyzed within a frequency bandwidth ranging only from LF to MF. To meet the design specifications that are needed to ensure satisfactory performance of the probe, the forecasts of the sensitivityfunctions approach are more reliable than the results foreseen by the transfer-functions method. In other words, once the measurement inaccuracy is bound by an acceptable limit $(10 \%)$, the sensitivity approach provides more realistic values with respect to those provided by the transfer method. These numeric values concern both the band of frequency $f$ for the probe and the measurable range of resistivity $\rho$ or permittivity $\varepsilon_{r}$ for the soils and concretes (the order of magnitude of these values is reported in the relevant literature; see Settimi et al. 2010, and references therein).

The present report (see also Earth-print.org and arXiv.org: Settimi et al. 2009) discusses the development and engineering of a suitable quadrupolar probe for simultaneous and noninvasive surveys of electrical resistivity and dielectric permittivity. The quadrupolar probe can perform measurements on a subsurface with inaccuracies below a fixed limit (10\%) in a bandwidth of LF $(100 \mathrm{kHz})$. The quadrupole should be connected to an appropriate ADC that can sample in phase and quadrature (IQ) [Jankovic and Öhman 2001] or in uniform mode. If the probe is 
characterized by galvanic contact with the surface, the inaccuracies in the measurement of the resistivity and permittivity due to the IQ or uniform sampling ADC are analytically expressed. A large number of numerical simulations shows that the performances of the probe depends on the selected sampler, and that the IQ is better, compared to the uniform mode, under the same operating conditions, i.e. for bit resolution and medium.

This report is organized as follows. Following this introductory Section 1, Section 2 defines the data acquisition system. In Section 3, the theoretical modeling is provided for both the IQ (3.1) and the uniform (3.2) samplers. In Section 4 , the design of the characteristic geometrical dimensions of the quadrupolar probe is analyzed. Section 5 proposes a concluding discussion. Finally, the Appendix provides an outline of the somewhat lengthy calculations.

\section{Data-acquisition system}

To design a quadrupole probe (Figure 1) that can measure the electrical conductivity $\sigma$ and the dielectric permittivity $\varepsilon_{r}$ of a subjacent medium with inaccuracies below a prefixed limit $(10 \%)$ in a band of low frequencies $(B=100 \mathrm{kHz})$, the probe needs to be connected to an appropriate ADC. Such an ADC should perform the IQ and uniform sampling [Razavi 1995, Jankovic and Öhman 2001] with bit resolution not exceeding 12 , thereby rendering the system of measurement (voltage scale of $4 \mathrm{~V}$ ) almost insensitive to the electrical noise of the external environment $(\approx 1 \mathrm{mV})$.

The IQ can be implemented using a technique that is easier to achieve than for the uniform mode, because the voltage signal of the probe is in the frequency band of $B=100$ $\mathrm{kHz}$ and the IQ is sampled with a rate of only $f_{S}=4 B=400$ $\mathrm{kHz}$, while, for example, low resolution uniform samplers are specified for rates of $5 \mathrm{MHz}$ to $200 \mathrm{MHz}$.

With the aim of investigating the physics of the measuring system, the inaccuracies in the transfer impedance measured by the quadrupolar probe due to uniform or IQ sampling ADCs are provided here.

For the stage downstream of the quadrupole, if the electrical voltage $V$ is amplified, $V_{V}=A_{V} V$, and the intensity of the current $I$ is transformed by a trans-resistance amplifier, $V_{I}=A_{R} I$, the signals having been processed by the sampler, then:

the inaccuracy $\Delta|Z| /|Z|$ for the modulus of the transfer impedance results from the negligible contributions of $\Delta A_{V} / A_{V}$ and $\Delta A_{R} / A_{R}$ for the voltage and trans-resistance amplifiers, respectively, and the predominant $\Delta\left|V_{V}\right| /\left|V_{V}\right|$ for the modulus of the voltage, due to the sampling,

$$
\frac{\Delta|Z|}{|Z|}=\frac{\Delta A_{V}}{A_{V}}+\frac{\Delta A_{R}}{A_{R}}+2 \frac{\Delta\left|V_{V}\right|}{\left|V_{V}\right|} \cong 2 \frac{\Delta\left|V_{V}\right|}{\left|V_{V}\right|}
$$

the inaccuracies for the modulus of the voltage and the current intensity being equal, $\Delta\left|V_{V}\right| /\left|V_{V}\right|=\Delta\left|V_{I}\right| /\left|V_{I}\right|$.
Instead, the inaccuracy $\Delta \Phi_{Z} / \Phi_{Z}$ for the initial phase of the transfer impedance coincides with the inaccuracy $\Delta \varphi_{V} / \varphi_{V}$ for the phase of the voltage, due to the sampler,

$$
\frac{\Delta \Phi_{Z}}{\Phi_{Z}}=\frac{\Delta \varphi_{V}}{\varphi_{V}}
$$

the initial phase of the current being zero, $\varphi_{I}=0$.

\section{Theoretical modeling}

Let us consider the IQ mode [Jankovic and Öhman 2001]. The IQ quartz is oscillating with a period $T$ that is affected by an inaccuracy $\Delta T / T$. The quartz figure of merit $Q=T / \Delta T$ assumes high enough values, i.e. $1 / Q<<1 /(2 \pi)$ $\left[Q=10^{4}-10^{6}\right]$. It can be shown that the inaccuracy $\Delta|Z| /|Z|_{I Q}\left(n, \varphi_{V}\right)$ depends strongly on the bit resolution $n$, decreasing as the power function $2^{-n}$ of $n$, and weakly on the initial phase of voltage $\varphi_{V}$, such that (see also comments below Equation 3.7):

$$
\begin{aligned}
& \left.\frac{\Delta|Z|}{|Z|}\right|_{I Q} \cong \frac{1}{2^{n}}\left[1+\frac{2 \pi}{Q} \tan \varphi_{V}\left(1+\cos 2 \varphi_{V}\right)\right]= \\
& =\left\{\begin{array}{l}
\frac{1}{2^{n}}\left(1+\frac{2 \pi}{Q}\right), \varphi_{V}=\varphi_{V}^{\max }=\frac{\pi}{4} \\
\frac{1}{2^{n}}\left(1-\frac{2 \pi}{Q}\right), \varphi_{V}=\varphi_{V}^{\min }=\pi-\varphi_{V}^{\max }
\end{array},\right.
\end{aligned}
$$

with $\Delta|Z| /|Z|_{I Q}\left(n, \varphi_{V}\right)$ being:

$$
\left.\lim _{\varphi_{V} \rightarrow \varphi_{V}^{\operatorname{im}}} \frac{\Delta|Z|}{|Z|}\right|_{I Q}=\frac{1}{2^{n}}
$$

in the limit value:

$$
\varphi_{V}^{\lim }=\operatorname{arctg}\left(\frac{Q}{4 \pi}\right) \approx \frac{\pi}{2} .
$$

Instead, for uniform sampling [Razavi 1995], the inaccuracy $\Delta|Z| /|Z|_{U}(n)$ for $|Z|$ only depends on the bit resolution $n$, decreasing as the power function $2^{-n}$ of $n$,

$$
\left.\frac{\Delta|Z|}{|Z|}\right|_{U}=\frac{1}{2^{n}}
$$

Consequently, for all ADCs with a bit resolution $n$ :

if the probe performs measurements on a medium, then the inaccuracy $\Delta \varepsilon_{r} / \varepsilon_{r}(f)$ in the measurement of the dielectric permittivity $\varepsilon_{r}$ is characterized by a minimum limit $\Delta \varepsilon_{r} /\left.\varepsilon_{r}\right|_{\min }\left(\varepsilon_{r}, n\right)$, which can be interpreted as the "physical bound» imposed on the inaccuracies of the problem, which depend on both $\varepsilon_{r}$ and the bit resolution $n$, as it is directly proportional to the factor $\left(1+1 / \varepsilon_{r}\right)$, while it decreases as the power function $2^{-n}$ of $n$ (Figure 2a);

if a probe with characteristic geometrical dimension $L$ performs measurements on a medium with conductivity $\sigma$ and permittivity $\varepsilon_{r}$, working within the cut-off frequency $f_{T}=f_{T}\left(\sigma, \varepsilon_{r}\right)=\sigma /\left(2 \pi \varepsilon_{0}\left(\varepsilon_{r}+1\right)\right.$ [Settimi et al. 2010], then the absolute error $E_{|Z|}(L, \sigma, n)$ in the measurement of the 


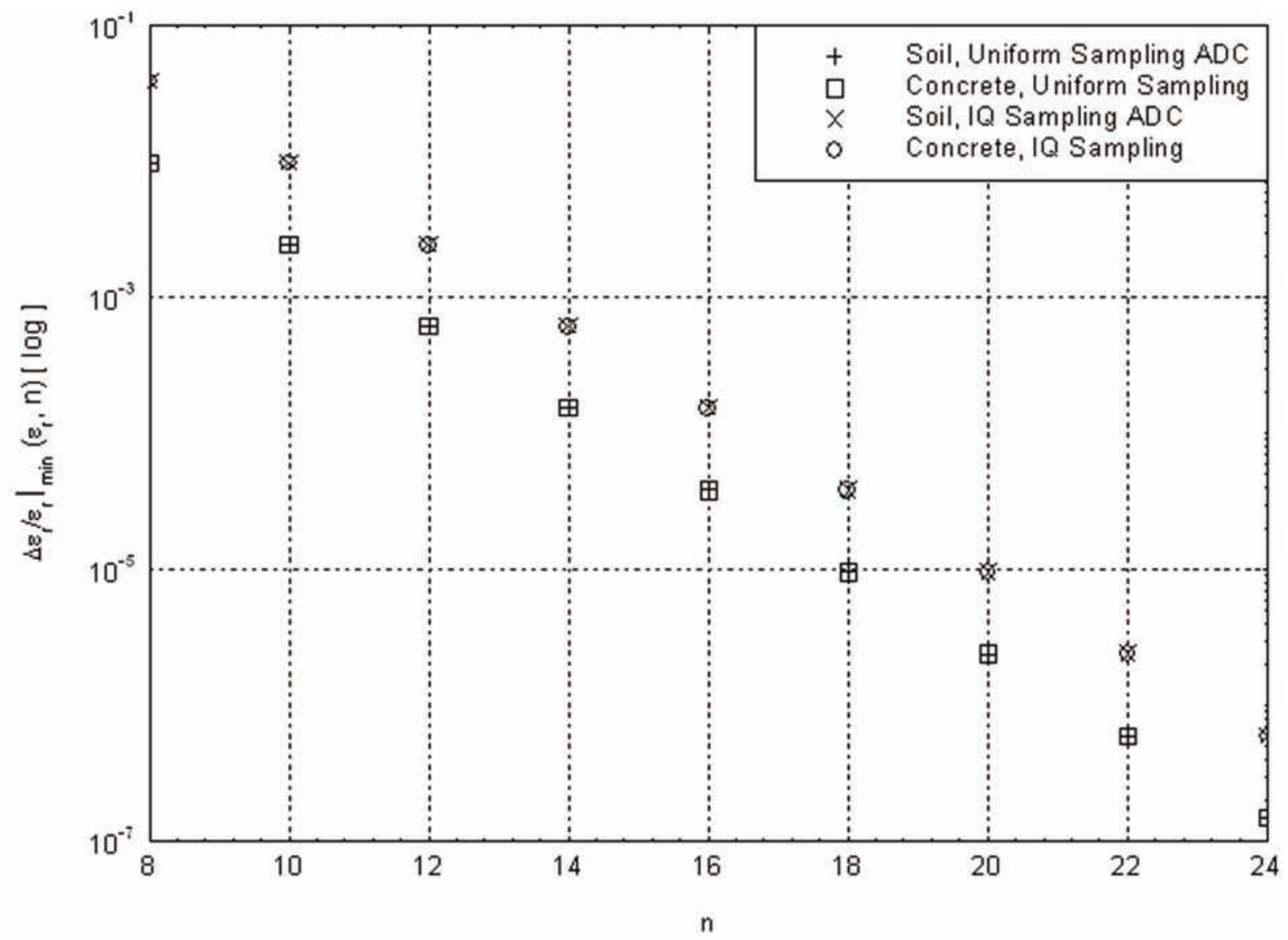

a)

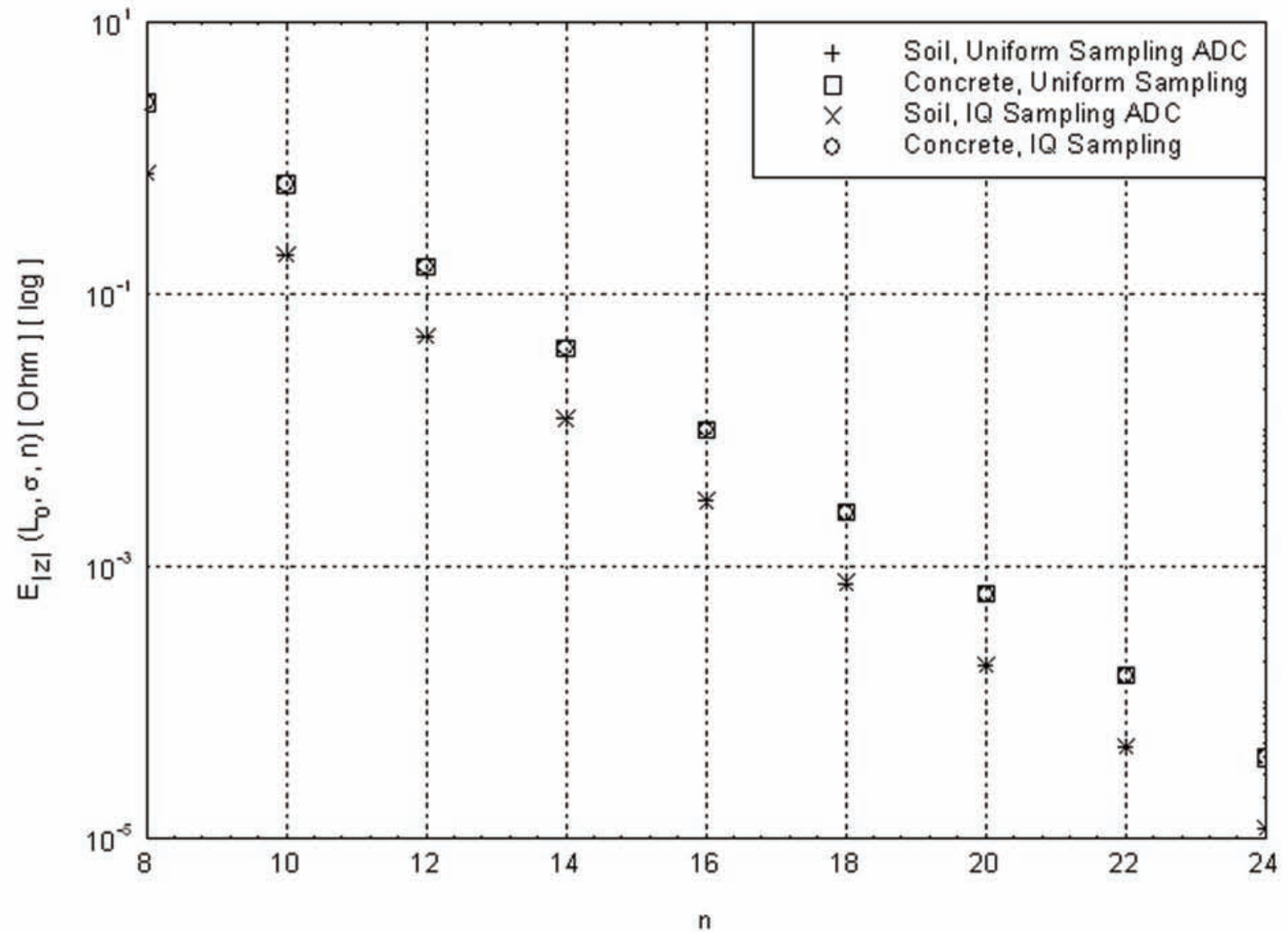

Figure 2. A quadrupole of characteristic geometrical dimension $L_{0}=1 \mathrm{~m}$ has galvanic contact on a subsurface of dielectric permittivity around $\varepsilon_{r} \approx 4$ and low electrical conductivity $\sigma$, like a nonsaturated terrestrial soil $\left(\sigma \approx 3.334 \cdot 10^{-4} \mathrm{~S} / \mathrm{m}\right)$ or concrete $\left(\sigma \approx 10^{-4} \mathrm{~S} / \mathrm{m}\right)$. With the hypothesis that the probe is connected to a sampler of bit resolution $n$, which ranges from 8 bit to 24 bit: (a) semi-logarithmic plot for the "physical bound» imposed on the inaccuracies, i.e. $\Delta \varepsilon_{r} /\left.\varepsilon_{r}\right|_{\text {min }}\left(\varepsilon_{r}, n\right)$, as a function of the resolution $n$; (b) semi-logarithmic plot for the absolute error $E_{|Z|}\left(L_{0}, \sigma, n\right)$ for the transfer impedance in modulus below its cut-off frequency, as a function of the resolution $n$. 


\begin{tabular}{|c|c|c|c|c|c|}
\hline \multirow{2}{*}{ Soil } & \multirow{2}{*}{$\begin{array}{l}\text { U Sampling ADC } \\
\text { (12 bit, } 10 \mathrm{MS} / \mathrm{s})\end{array}$} & \multicolumn{3}{|c|}{ U Sampling } & \multirow{3}{*}{$\begin{array}{c}\text { IQ Sampling } \\
\text { (12 bit) }\end{array}$} \\
\hline & & (12 bit, $60 \mathrm{MS} / \mathrm{s})$ & (12 bit, $200 \mathrm{MS} / \mathrm{s}$ ) & & \\
\hline \multicolumn{5}{|c|}{$\left(\varepsilon_{r}=4, \rho=3000 \Omega \cdot m\right)$} & \\
\hline$f_{\text {opt }}$ & $150.361 \mathrm{kHz}$ & $267.569 \mathrm{kHz}$ & $387.772 \mathrm{kHz}$ & & $\approx 1.459 \mathrm{MHz}$ \\
\hline$f_{\min }$ & $92.198 \mathrm{kHz}$ & $98.042 \mathrm{kHz}$ & $95.055 \mathrm{kHz}$ & & $94.228 \mathrm{kHz}$ \\
\hline$f_{\max }$ & $265.287 \mathrm{kHz}$ & $885.632 \mathrm{kHz}$ & $\approx 1.832 \mathrm{MHz}$ & & $\approx 16.224 \mathrm{MHz}$ \\
\hline Concrete & (8 bit, $50 \mathrm{MS} / \mathrm{s})$ & (8 bit, $100 \mathrm{MS} / \mathrm{s})$ & $(8 \mathrm{bit}, 250 \mathrm{MS} / \mathrm{s})$ & (8 bit, 2 GS/s) & \\
\hline \multicolumn{6}{|c|}{$\left(\varepsilon_{r}=4, \rho=10000 \Omega \cdot m\right)$} \\
\hline$f_{\text {opt }}$ & $241.41 \mathrm{kHz}$ & $289.103 \mathrm{kHz}$ & $364.485 \mathrm{kHz}$ & $607.522 \mathrm{kHz}$ & \\
\hline$f_{\min }$ & $98.574 \mathrm{kHz}$ & $96.629 \mathrm{kHz}$ & $95.553 \mathrm{kHz}$ & $94.953 \mathrm{kHz}$ & \\
\hline$f_{\max }$ & $591.657 \mathrm{kHz}$ & $815.1 \mathrm{kHz}$ & $\approx 1.079 \mathrm{MHz}$ & $\approx 1.443 \mathrm{MHz}$ & \\
\hline Concrete & (12 bit, $5 \mathrm{MS} / \mathrm{s}$ ) & $(12 \mathrm{bit}, 10 \mathrm{MS} / \mathrm{s})$ & $(12 \mathrm{bit}, 60 \mathrm{MS} / \mathrm{s})$ & (12 bit, $200 \mathrm{MS} / \mathrm{s})$ & (12 bit) \\
\hline \multicolumn{6}{|c|}{$\left(\varepsilon_{r}=4, \rho=10000 \Omega \cdot m\right)$} \\
\hline$f_{o p t}$ & $53.271 \mathrm{kHz}$ & $66.605 \mathrm{kHz}$ & $116.332 \mathrm{kHz}$ & $165.329 \mathrm{kHz}$ & $437.716 \mathrm{kHz}$ \\
\hline$f_{\min }$ & $35.085 \mathrm{kHz}$ & $30.646 \mathrm{kHz}$ & $28.517 \mathrm{kHz}$ & $28.273 \mathrm{kHz}$ & $28.268 \mathrm{kHz}$ \\
\hline$f_{\max }^{\min }$ & $85.396 \mathrm{kHz}$ & $169.271 \mathrm{kHz}$ & $549.595 \mathrm{kHz}$ & $\approx 1.016 \mathrm{MHz}$ & $\approx 4.867 \mathrm{MHz}$ \\
\hline
\end{tabular}

Table 1. With reference to the caption of Figure 3: a quadrupolar probe is connected to a uniform or IQ ADC (bit resolution $n$ ). Data are shown for the optimal working frequency, $f_{\text {opt }}$, that minimizes the inaccuracy in the measurement of permittivity, $\Delta \varepsilon_{r} / \varepsilon_{r}(f)$, minimum and maximum frequencies, $f_{\min }$ and $f_{\max }$, that limit the inaccuracies of permittivity and conductivity, $\Delta \varepsilon_{r} / \varepsilon_{r}(f) \leq 0.1$ and $\Delta \sigma / \sigma(f) \leq 0.1$, for measurements performed on soil, with $n=12$, and on concrete, with $n=8$ and $n=12$.

modulus for the transfer impedance $|Z|_{N}(L, \sigma)$ depends on $\sigma$, $L$ and the bit resolution $n$, with the error inversely proportional to both $\sigma$ and $L$, while it decreases as the power function $2^{-n}$ of $n$ (Figure 2b).

Consider an IQ mode [Jankovic and Öhman 2001]. It can be shown that the inaccuracy $\Delta \Phi_{Z} /\left.\Phi_{Z}\right|_{\text {IQ }}\left(n, \varphi_{V}\right)$ depends both on the bit resolution $n$, decreasing as the power function $2^{-n}$ of $n$, and on the voltage phase $\varphi_{V}$, such that (see also comments below Equation 3.7):

$$
\begin{aligned}
\left.\frac{\Delta \Phi_{Z}}{\Phi_{Z}}\right|_{I Q} \cong \frac{1}{2^{n}} \frac{\sin \left(2 \varphi_{V}\right)}{2 \varphi_{V}}\left(1+\frac{2 \pi}{Q} \tan \varphi_{V}\right)= \\
\quad=\left\{\begin{array}{ll}
\frac{1}{2^{n}}, & \varphi_{V}=\varphi_{V}^{\max }=0 \\
0, & \varphi_{V}=\varphi_{V}^{\min }=\pi
\end{array},\right.
\end{aligned}
$$

with $\Delta \Phi_{Z} /\left.\Phi_{Z}\right|_{I Q}\left(n, \varphi_{V}\right)$ being very low in $\varphi_{V}=\pi / 2$, due to the high $Q$,

$$
\left.\lim _{\varphi_{V} \rightarrow \pi / 2} \frac{\Delta \Phi_{Z}}{\Phi_{Z}}\right|_{I Q}=\frac{1}{2^{n-2}} \frac{1}{Q} .
$$

Instead, for uniform sampling, the inaccuracy $\Delta \Phi_{Z} /\left.\Phi_{Z}\right|_{U}$ $\left(f, f_{S}\right)$ for $\Phi_{Z}$ depends on both the working frequency $f$ of the probe and the rate sampling $f_{S}$ of the ADC, with the inaccuracy directly proportional to the frequency ratio $f / f_{S}$,

$$
\left.\frac{\Delta \Phi_{Z}}{\Phi_{Z}}\right|_{U}=2 \frac{f}{f_{S}} .
$$

As a consequence, and only for uniform sampling ADCs, the inaccuracy $\Delta \Phi_{Z} / \Phi_{Z}\left(f, f_{S}\right)$ for the phase $\Phi_{Z}$ must be optimized in the upper frequency $f_{u p}$, so when the probe performs measurements at the limit of its band $B, f_{u p}=B$.
Let us provide some clues with respect the derivation of Equations (3.1) and (3.5). Ideally, a sinusoidal signal $V(t)=$ $V_{0} \cdot \sin \left(\omega t+\varphi_{V}\right)$ with an angular frequency $\omega=2 \pi / T$ and a period $T$ can be sampled by an IQ mode. The in-phase $V_{I}=$ $V(t=0)=V_{0} \cdot \sin \left(\varphi_{V}\right)$ and quadrature $V_{Q}=V(t=T / 4)=$ $V_{0} \cdot \cos \left(\varphi_{V}\right)$ values are obtained. The amplitude $V_{0}$ and phase $\varphi_{V}$ of the sinusoidal signal are linked to the IQ values. Indeed: $V_{0}=\left[\left(V_{I}\right)^{2}+\left(V_{Q}\right)^{2}\right]^{1 / 2}$ and $\varphi=\operatorname{arctg}\left(V_{I} / V_{Q}\right)$. The inaccuracies of the IQ values $\Delta V_{I} / V_{I}=\Delta V_{Q} / V_{Q}=1 / 2^{n+1}$ is defined by the bit resolution $n$ of the IQ sampling. Applying the theory of error propagation, the inaccuracies of the amplitude $V_{0}$ and phase $\varphi_{V}$ are derived. Indeed: $\Delta V_{0} / V_{0}=1 / 2^{n}$ and $\Delta \varphi_{V} / \varphi_{V}=$ $1 / 2^{n} \cdot\left[\sin \left(\varphi_{V}\right) / \varphi_{V}\right]$. More realistically, the quartz internal to the IQ sampler is oscillating with a period $T$ that is affected by an inaccuracy $\Delta T / T$. Suppose that the merit figure of quartz $Q=T / \Delta T$ assumes high enough values, i.e. $1 / Q<<$ $1 /(2 \pi)$. The Taylor's series expansion to first order in $1 / Q$ better approximates the values actually sampled, and in particular, the quadrature value $V_{Q, \Delta T}=V(t=T / 4+\Delta T)$, and consequently the amplitude $V_{Q, \Delta T}=\left[\left(V_{I}\right)^{2}+\left(V_{Q, \Delta T}\right)^{2}\right]^{1 / 2}$ and phase $\varphi_{\Delta T}=\operatorname{arctg}\left(V_{I} / V_{Q, \Delta T}\right)$ of the sinusoidal signal. Indeed: $V_{Q, \Delta T} \approx V_{Q}-V_{I} \cdot(2 \pi / Q), V_{0, \Delta T} \approx V_{0} \cdot\left[1-(\pi / Q) \cdot \sin \left(2 \varphi_{V}\right)\right]$ and $\operatorname{tg} \varphi_{\Delta T} \approx \operatorname{tg}\left(\varphi_{V}\right) \cdot\left[1+(2 \pi / Q) \cdot \operatorname{tg}\left(\varphi_{V}\right)\right]$. Moreover, the inaccuracy $\Delta T / T$ relative to the quartz merit figure $Q=1 /(\Delta T / T)$ increases the inaccuracy of the quadrature value to $\Delta V_{Q, \Delta T} / V_{Q, \Delta T} \approx \Delta V_{Q} / V_{Q} \cdot\left[1+(4 \pi / Q) \cdot \operatorname{tg}\left(\varphi_{V}\right)\right]$. Finally, in applying again the theory of error propagation, the inaccuracies of the amplitude $V_{0, \Delta T}$ and phase $\varphi_{V, \Delta T}$ are now derived in the case of a nonideal IQ quartz. So, some measurement inaccuracies similar to Equations (3.1) and (3.5) are derived: $\Delta V_{0, \Delta T} / V_{0, \Delta T} \approx \Delta V_{0} / V_{0} \cdot\left[1+(2 \pi / Q) \cdot \operatorname{tg}\left(\varphi_{V}\right)(1+\right.$ $\left.\left.2 \cos \left(2 \varphi_{V}\right)\right)\right]$ and $\Delta \varphi_{V, \Delta T} / \varphi_{V, \Delta T} \approx \Delta \varphi_{V} / \varphi_{V}\left[1+(2 \pi / Q) \cdot \operatorname{tg}\left(\varphi_{V}\right)\right]$. 
SOIL

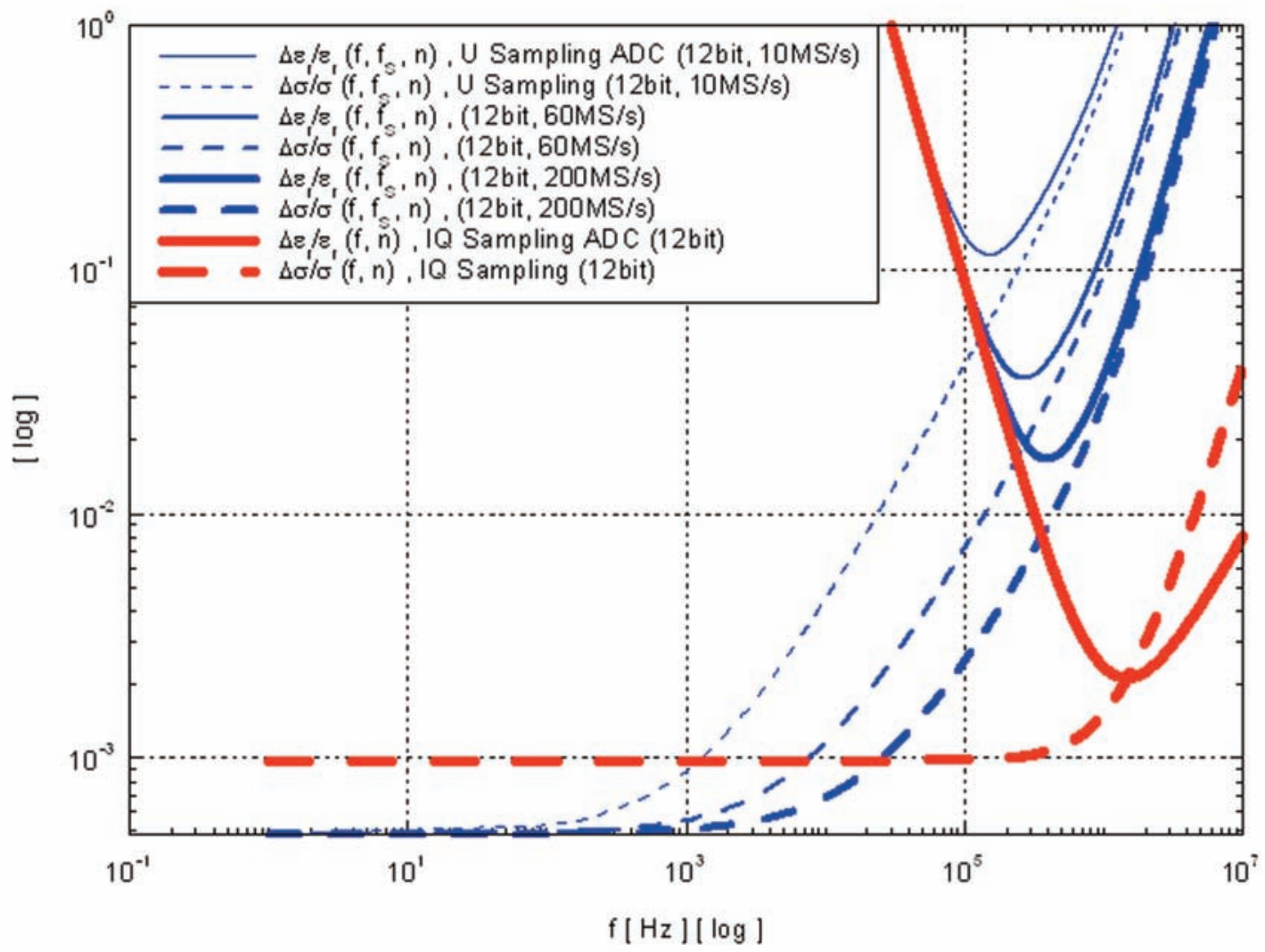

a)

CONCRETE

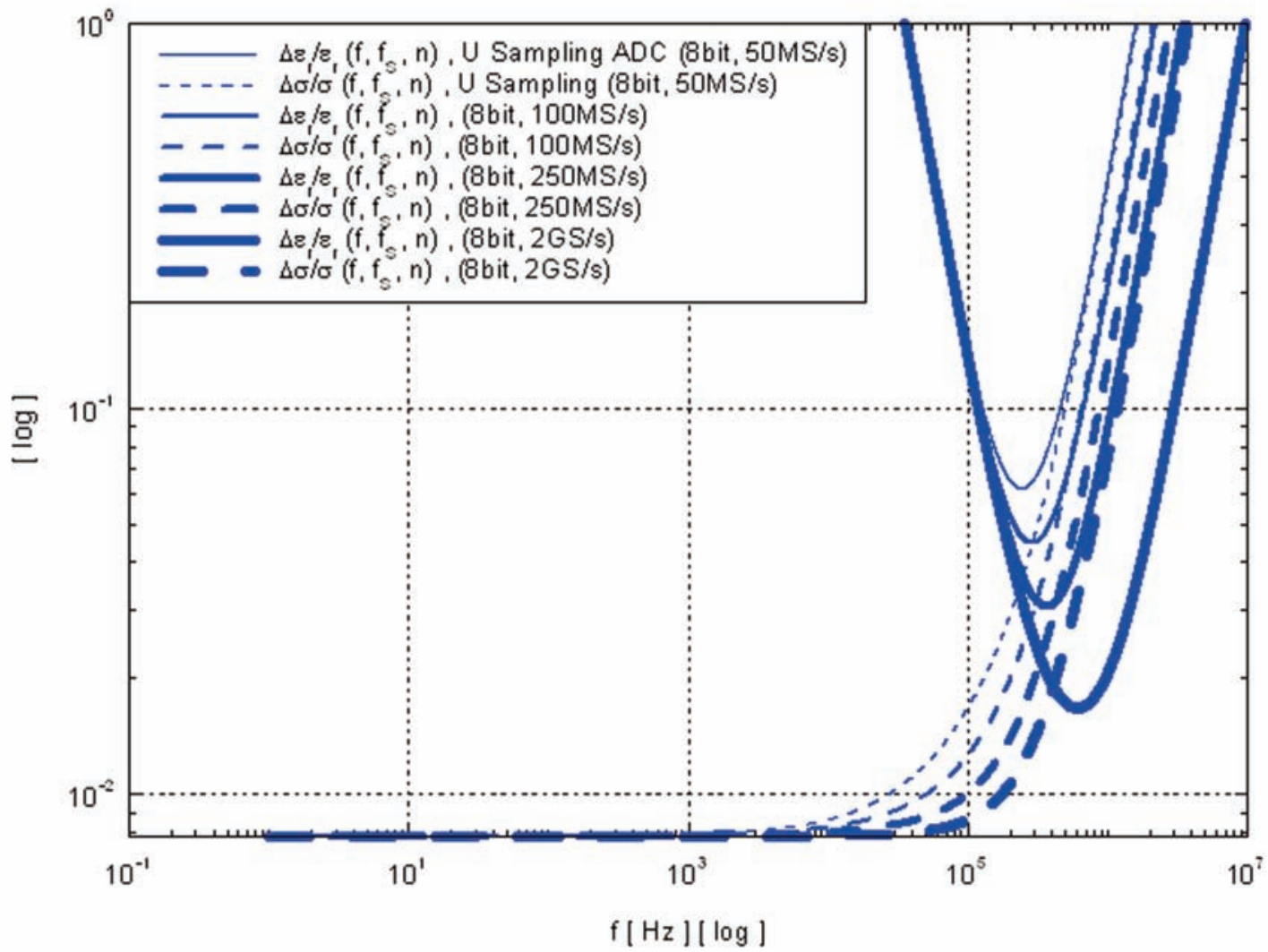

b)

Figure 3 (this page and the next). With reference to the operating conditions described in the caption to Figure 2 (for quadrupole and subsurfaces). Bode's diagrams for the inaccuracies $\Delta \varepsilon_{r} / \varepsilon_{r}(f)$ and $\Delta \sigma / \sigma(f)$ in the measurements of the dielectric permittivity $\varepsilon_{r}$ and the electrical conductivity $\sigma$, plotted as functions of the frequency $f$, for nonsaturated terrestrial soil (a) and concrete (b, c) analyses. The probe is connected to uniform or IQ or samplers (in the worst case, when the internal quartz is oscillating at its lowest merit factor $Q \approx 104$ ), of bit resolution $n=12$ (a, c) or $n=8$ (b), which allow inaccuracies in the measurements below a prefixed limit, of $15 \%(\mathrm{a}, \mathrm{b})$ and $10 \%(\mathrm{a}, \mathrm{c})$, within the frequency band $B=100 \mathrm{kHz}$ (Table 1 ). 


\section{CONCRETE}

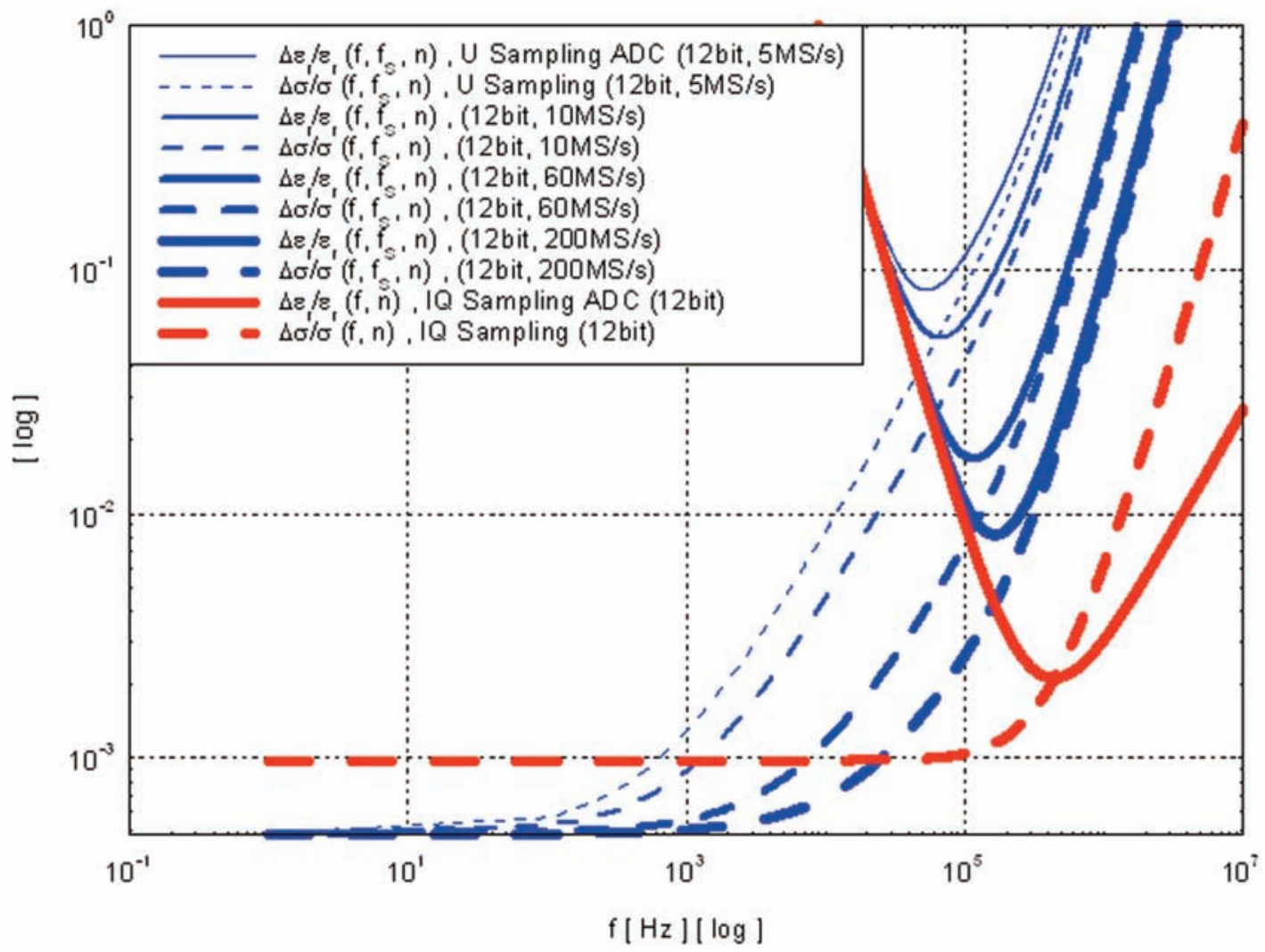

c)

Continuing with the aim of investigating the physics of the measuring system, when the quadrupolar probe shows galvanic contact with the subjacent medium of electrical conductivity $\sigma$ and dielectric permittivity $\varepsilon_{r}$, i.e. $h=0$, and works in frequencies $f$ lower than the cut-off frequency $f_{T}=$ $f_{T}\left(\sigma, \varepsilon_{r}\right)$ [Grard and Tabbagh 1991],

$$
\Omega=\frac{\omega}{\omega_{T}} \leq 1,
$$

the inaccuracies $\Delta \sigma / \sigma$ in the measurements of the conductivity $\sigma$ and $\Delta \varepsilon_{r} / \varepsilon_{r}$ for the permittivity $\varepsilon_{r}$ are analytically expressed (Figure 3; Table 1), and achievable connecting IQ or uniform samplers, which ensure the inaccuracies $\Delta|Z| /|Z|$ for the modulus $|Z|$, and $\Delta \Phi_{Z} / \Phi_{Z}$ for the phase $\Phi_{Z}$ of the transfer impedance [Settimi et al. 2010],

$$
\begin{array}{r}
\frac{\Delta \sigma}{\sigma} \cong 2\left(1+\Omega^{2}\right)\left(\frac{\Delta|Z|}{|Z|}+\frac{\Delta \Phi_{Z}}{\Phi_{Z}}\right), \\
\frac{\Delta \varepsilon_{r}}{\varepsilon_{r}} \cong 2\left(1+\Omega^{2}\right)\left(1+\frac{1}{\varepsilon_{r}}\right)\left(\frac{1}{\Omega^{2}} \frac{\Delta|Z|}{|Z|}+\frac{\Delta \Phi_{Z}}{\Phi_{Z}}\right),
\end{array}
$$

Only if the quadrupole probe is in galvanic contact with the subjacent medium, i.e. $h=0$, then our mathematicalphysical model predicts that the inaccuracies $\Delta \sigma / \sigma$ for $\sigma$ and $\Delta \varepsilon_{r} / \varepsilon_{r}$ for $\varepsilon_{r}$ are invariant in the linear (Wenner's) or square configuration, and independent of the characteristic geometrical dimension of the quadrupole, i.e. the electrodeelectrode distance $L$ [Settimi et al. 2010]. If besides grazing the medium, the quadrupole measures $\sigma$ and $\varepsilon_{r}$ working in a frequency $f$ that is much lower than the cut-off frequency $f_{T}=f_{T}\left(\sigma, \varepsilon_{r}\right)$, then the inaccuracy $\Delta \sigma / \sigma=F(\Delta|Z| /|Z|$, $\left.\Delta \Phi_{Z} / \Phi_{Z}\right)$ is a linear combination of the inaccuracies $\Delta|Z| /|Z|$ and $\Delta \Phi_{Z} / \Phi_{Z}$ for the transfer impedance, while the inaccuracy $\Delta \varepsilon_{r} / \varepsilon_{r}=F(\Delta|Z| /|Z|)$ can be approximated as a linear function only of the inaccuracy $\Delta|Z| /|Z|$; in other words, if $f<<f_{T}$, then $\Delta \Phi_{Z} / \Phi_{Z}$ is contributing in $\Delta \sigma / \sigma$ but not in $\Delta \varepsilon_{r} / \varepsilon_{r}$.

\subsection{For IQ sampling}

Let us consider IQ sampling ADCs (merit figure $Q$ ) [Jankovic and Öhman 2001]. The inaccuracy $\left.\Delta \varepsilon_{r} / \varepsilon_{r} f f, n\right)$ in the measurement of permittivity $\varepsilon_{r}$ is characterized by an optimal working frequency $f_{\text {opt,IQ }}\left(f_{T}\right)$, which is close to the cut-off frequency for the modulus of the transfer impedance $f_{T}=f_{T}\left(\sigma, \varepsilon_{r}\right)$, i.e.

$$
f_{\text {opt }, I Q} \cong f_{T}\left(1+\frac{\pi / 2}{Q}\right) \approx f_{T} .
$$

This optimal frequency is tuned in a minimum value of inaccuracy $\Delta \varepsilon_{r} /\left.\varepsilon_{r}\right|_{\min , I Q}\left(\varepsilon_{r}, n\right)$, which depends both on $\varepsilon_{r}$ and on the specifications of the sampler, and in particular, its bit resolution $n$ only. The minimum value of inaccuracy is directly proportional to the factor $\left(1+1 / \varepsilon_{r}\right)$, while decreasing as the power function $2^{-(n-3)}$ of $n$, such that (Figure 2a):

$$
\left.\frac{\Delta \varepsilon_{r}}{\varepsilon_{r}}\right|_{\text {min, }, \mathrm{Q}} \cong\left(1+\frac{1}{\varepsilon_{r}}\right) \frac{1}{2^{n-3}}\left(1+\frac{\pi}{Q}\right) .
$$



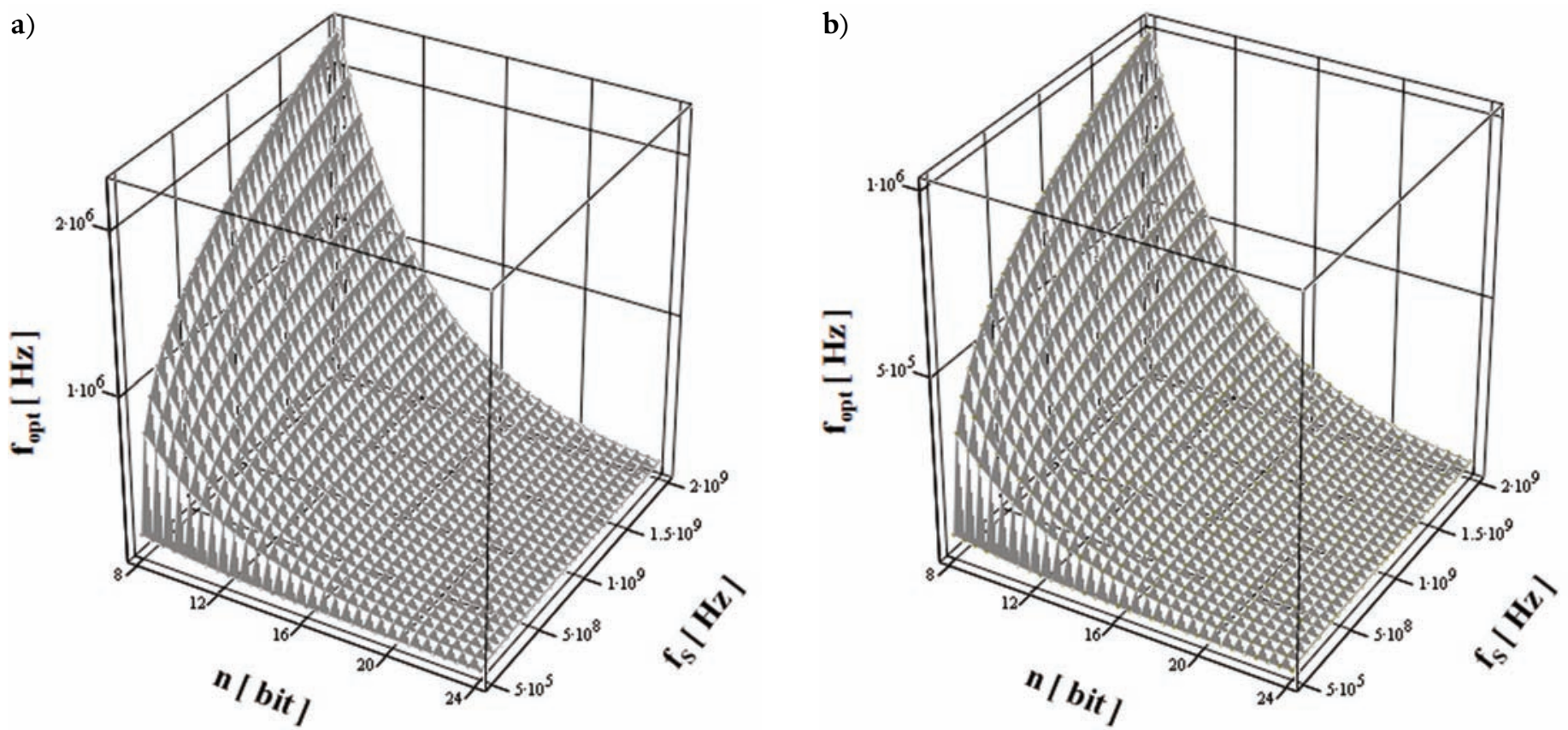

Figure 4. With reference to the operating conditions described in the caption to Figure 2 (for quadrupolar probe). Plots for the optimal working frequency $f_{o p t, U}\left(n, f_{S}\right)$, which minimizes the inaccuracies in the measurements of dielectric permittivity, as functions of both the bit resolution $n$ and the sampling rate $f_{S}$, for terrestrial soil (a) and concrete (b) analyses.

Consequently, if the frequency $f$ of the probe is much lower than the cut-off frequency $f_{T}\left(\sigma, \varepsilon_{r}\right)$ for the transfer impedance, then the inaccuracy $\Delta \sigma / \sigma(n)$ for $\sigma$ is a constant, and the inaccuracy $\Delta \varepsilon_{r} / \varepsilon_{r}(f, n)$ for $\varepsilon_{r}$ shows a downward trend in frequency, as $\left(f_{T} / f\right)^{2}$. Both these inaccuracies decrease as power functions of $n$, the first inaccuracy as $1 / 2^{n-2}$ and the second inaccuracy as $1 / 2^{n-1}$, i.e. (Figure 3; Table 1):

$$
\begin{gathered}
\left.\frac{\Delta \sigma}{\sigma}\right|_{I Q} \cong \frac{1}{2^{n-2}}\left(1+\frac{\pi}{Q}\right), \text { for } f<<f_{T}, \quad \text { (3.13) } \\
\left.\frac{\Delta \varepsilon_{r}}{\varepsilon_{r}}\right|_{I Q} \cong\left(1+\frac{1}{\varepsilon_{r}}\right) \frac{1}{2^{n-1}}\left(1+\frac{2 \pi}{Q}\right)\left[\frac{f_{T}}{f}\right]^{2}, \text { for } f<<f_{T} .
\end{gathered}
$$

Even if the frequency $f$ is much higher than $f_{T}\left(\sigma, \varepsilon_{r}\right)$, it can be shown that the inaccuracies $\Delta \sigma / \sigma(f, n)$ and $\Delta \varepsilon_{r} / \varepsilon_{r}(f, n)$ do not deviate too much from an upward trend in frequency, as the parabolic curve $\left(f / f_{T}\right)^{2}$ (see also comments below Equations 3.15 and 3.16). The parable gradient is high for the $\sigma$ measurement and low for the $\varepsilon_{r}$ measurement. Indeed, both of these inaccuracies still decrease as power functions of $n$. The first inaccuracy decreases as $1 / 2^{n-2}$ and the second inaccuracy as $1 / 2^{n-1}$ (Figure 3; Table 1), i.e.:

$$
\begin{aligned}
& \left.\frac{\Delta \sigma}{\sigma}\right|_{\mathrm{IQ}} \cong \frac{1}{2^{n-2}}\left(1+\frac{\pi}{Q}\right)\left[\frac{f}{f_{T}}\right]^{2}, \text { for } f>>f_{T}, \\
& \left.\frac{\Delta \varepsilon_{r}}{\varepsilon_{r}}\right|_{\mathrm{IQ}} \cong\left(1+\frac{1}{\varepsilon_{r}}\right) \frac{1}{2^{n-1}}\left[\frac{f}{f_{T}}\right]^{2} \text {, for } f>>f_{T} .
\end{aligned}
$$

A Mathcad program was run for the processing of some realistic values of the conductivity $\sigma$ and permittivity $\varepsilon_{r}$ for terrestrial soils and concretes. Mathcad numerically simulated the inaccuracies in the measurements of $\sigma$ and $\varepsilon_{r}$. The first pair of processed inaccuracies $\left(\Delta \sigma / \sigma, \Delta \varepsilon_{r} / \varepsilon_{r}\right)$ consisted of the exact "complex" expressions reported by Settimi et al. [2010], which hold for both LF and MF. The second pair of processed inaccuracies consists of the "simplified" Equations (3.9) and (3.10). As a first approximation, Equations (3.9) and (3.10) hold exactly for $\operatorname{LFs}\left(f<f_{T}\right)$, but not for MFs $\left(f>f_{T}\right)$. Indeed, the program has shown that the numerical curves of Settimi et al. [2010] overlap with the analytical curves of Equations (3.9) and (3.10). This overlap is excellent for $f<f_{T}$ and remains fine even for $f>f_{T}$.

Only if $f$ is lower than $f_{T}$ can the measurements then be optimized for $\varepsilon_{r}$ and $\sigma$. A requirement might be that the inaccuracy $\Delta \varepsilon_{r} / \varepsilon_{r}(n, f)$ for $\varepsilon_{r}$ is below the prefixed limit $\Delta \varepsilon_{r} /\left.\varepsilon_{r}\right|_{\text {fixed }}(10 \%)$ within the frequency band $B=100 \mathrm{kHz}$. A minimum bit resolution $n_{\min , I Q}\left(f_{T}, \varepsilon_{r}\right)$ should be chosen. This minimum bit resolution depends on both $f_{T}$ and $\varepsilon_{r}$ and increases as the logarithmic function $\log _{2}$ of both of the ratios $f_{T} / B$ and $1 / \varepsilon_{r}$, i.e.:

$$
n_{\text {min, IQ }} \approx 1-\left.\log _{2} \frac{\Delta \varepsilon_{r}}{\varepsilon_{r}}\right|_{\text {fixed }}+2 \log _{2} \frac{f_{T}}{B}+\log _{2}\left(1+\frac{1}{\varepsilon_{r}}\right) .
$$

Referring to the IQ sampling ADCs, the inaccuracies $\Delta \sigma / \sigma$ in the measurement of the electrical conductivity $\sigma$ and $\Delta \varepsilon_{r} / \varepsilon_{r}$ for the dielectric permittivity $\varepsilon_{r}$ were estimated for the worst case. So, the inaccuracies $\Delta|Z| /|Z|_{I_{I}(}\left(n, \varphi_{V}\right)$ in the measurement of the modulus and $\Delta \Phi_{Z} /\left.\Phi_{Z}\right|_{I Q}\left(n, \varphi_{V}\right)$ for the phase of the transfer impedance assume the mean and the maximum values, respectively; i.e. $\Delta|Z| /|Z|_{\text {IQ }}=$ $\Delta \Phi_{Z} /\left.\Phi_{Z}\right|_{\text {IQ }}=1 / 2^{n}$.

To conclude, for the IQ mode, the optimal and 


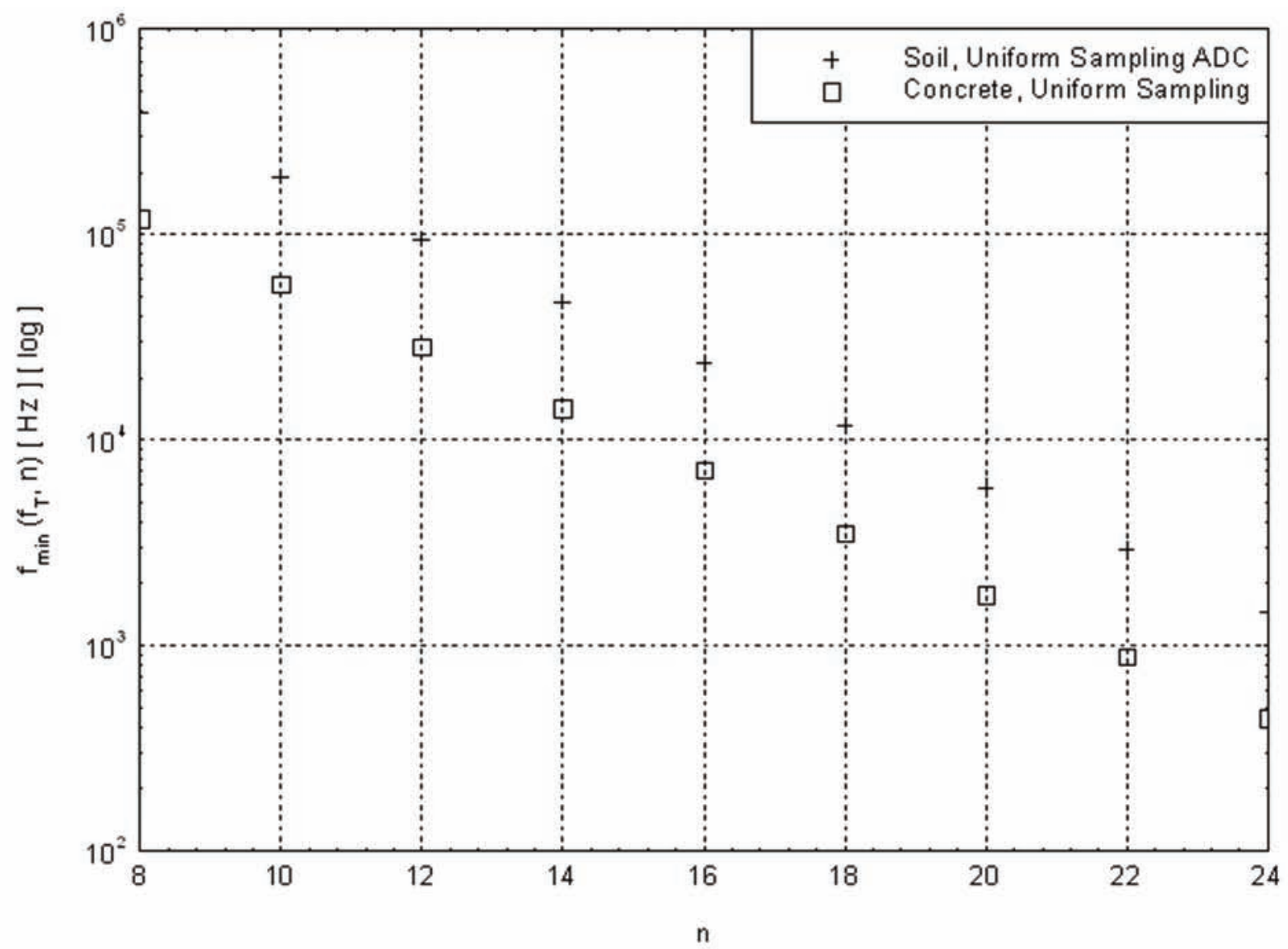

Figure 5. With reference to the operating conditions described in the caption to Figure 2 (for subsurface: nonsaturated terrestrial soil or concrete; sampling: uniform). Plot for the minimum values of frequency $f_{U, m i n}\left(n, f_{T}\right)$ that allow inaccuracy in the measurement of the dielectric permittivity below a prefixed limit $(10 \%)$, as a function of the bit resolution $n$ [the cut-off frequency of the transfer impedance fixed as $f_{T}=f_{T}\left(\varepsilon_{r}, \sigma\right)$, corresponding to the subsurface defined by the measurements $\left.\left(\varepsilon_{r}, \sigma\right)\right]$.

minimum values of the working frequency of the quadrupolar probe interact in a competitive way. The more the quadrupole analyzes a subjacent medium characterized by a low electrical conductivity, with the aim of shifting the optimal frequency into a low band, the more the low conductivity has the self-defeating effect of shifting the minimum value of frequency into a higher band. Indeed, the probe can work around a low optimal frequency, which is achievable in measurements of transfer impedance with a low cut-off frequency, as is typical of materials characterized by low conductivity. Instead, the more the minimum value of the working frequency is shifted into a lower band, the more the minimum bit resolution for the sampler has to be increased. If the medium was selected, then increasing the inaccuracy for the measurement of the dielectric permittivity, or, if the inaccuracy was fixed, shifting the cutoff frequency into a higher band, i.e. selecting a medium with high conductivity. Finally, our analysis in the present report shows that the quadrupole can work at a low optimal frequency if the transfer impedance is characterized by a low cut-off frequency, which is in agreement with more traditional results in the literature [Grard 1990a, Grard 1990b, Grard and Tabbagh 1991, Tabbagh et al. 1993, Vannaroni et al. 2004, Del Vento and Vannaroni 2005]. However, the probe can perform measurements in an appropriate band of higher frequencies centered around the cut-off frequency where the inaccuracy for the measurements of conductivity and permittivity were below a prefixed limit (10\%).

\subsection{For uniform sampling}

Let us consider uniform sampling ADCs [Razavi 1995]. Suppose that the frequency $f$ of the probe is lower than the cut-off frequency $f_{T}$ for the modulus $|Z|(f, L)$ of the transfer impedance, i.e. $f_{T}=f_{T}\left(\sigma, \varepsilon_{r}\right)$. It can be shown that the higher the bit resolution $n$, the more the optimal working frequency $f_{\text {opt }, U}\left(f_{T}, f_{S}, n\right)$ approximately depends on the cut-off frequency $f_{T}\left(\sigma, \varepsilon_{r}\right)$. The optimal frequency $f_{\text {opt }, U}\left(f_{T}, f_{S}, n\right)$ minimizes the inaccuracy $\Delta \varepsilon_{r} / \varepsilon_{r}\left(f, f_{S}, n\right)$ in the measurement of the permittivity $\varepsilon_{r}$. Moreover, this optimal frequency depends on the specifications of the sampler, and in particular the sampling rate $f_{S}$ and $n$. Finally, the frequency $f_{\text {opt }, U}\left(f_{T}, f_{S}, n\right)$ increases with both $f_{T}$ and $f_{S}$, while decreasing as the power function $2^{-n / 3}$ of $n$, such that (Figure 4):

$$
\frac{f_{o p t, U}}{f_{T}} \cong \sqrt[3]{\frac{1}{2^{n}} \frac{f_{S}}{f_{T}}} .
$$

Moreover, the higher the bit resolution $n$, the more the inaccuracy $\Delta \varepsilon_{r} / \varepsilon_{r}\left(f, f_{S}, n\right)$ for $\varepsilon_{r}$ cannot go down below a 


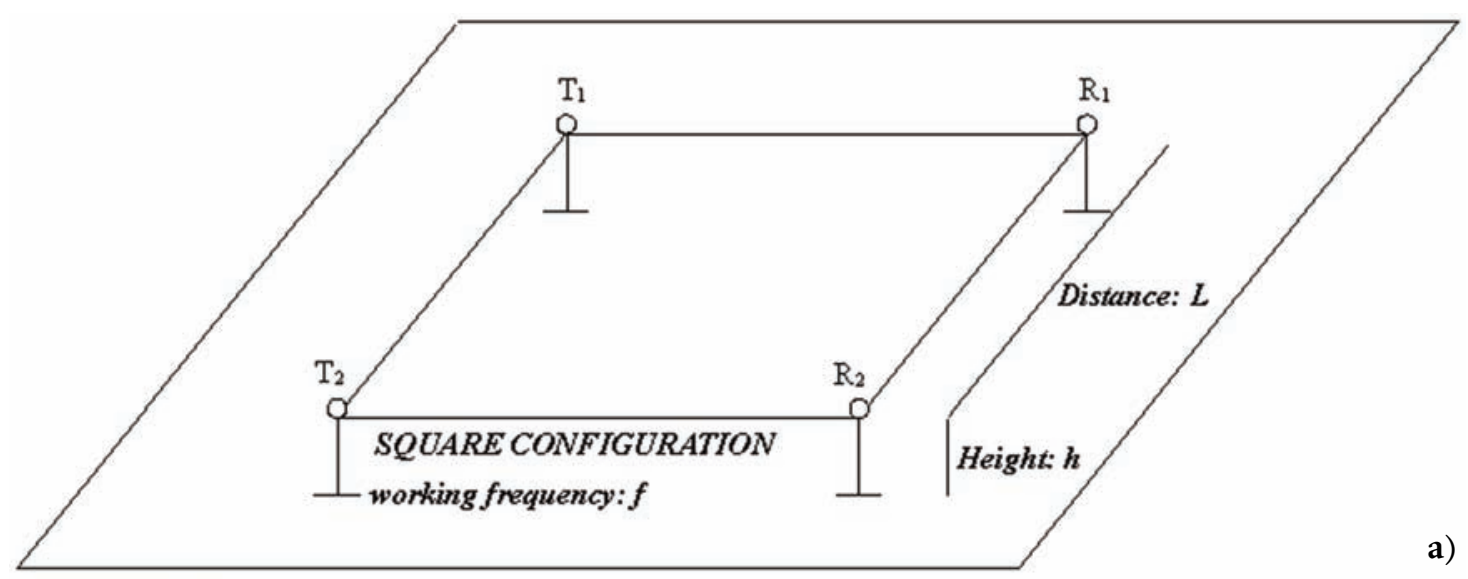

SUBSURFACE

Electrical Conductivity: $\sigma$

Dielectric Permittivity: $\varepsilon_{r}$

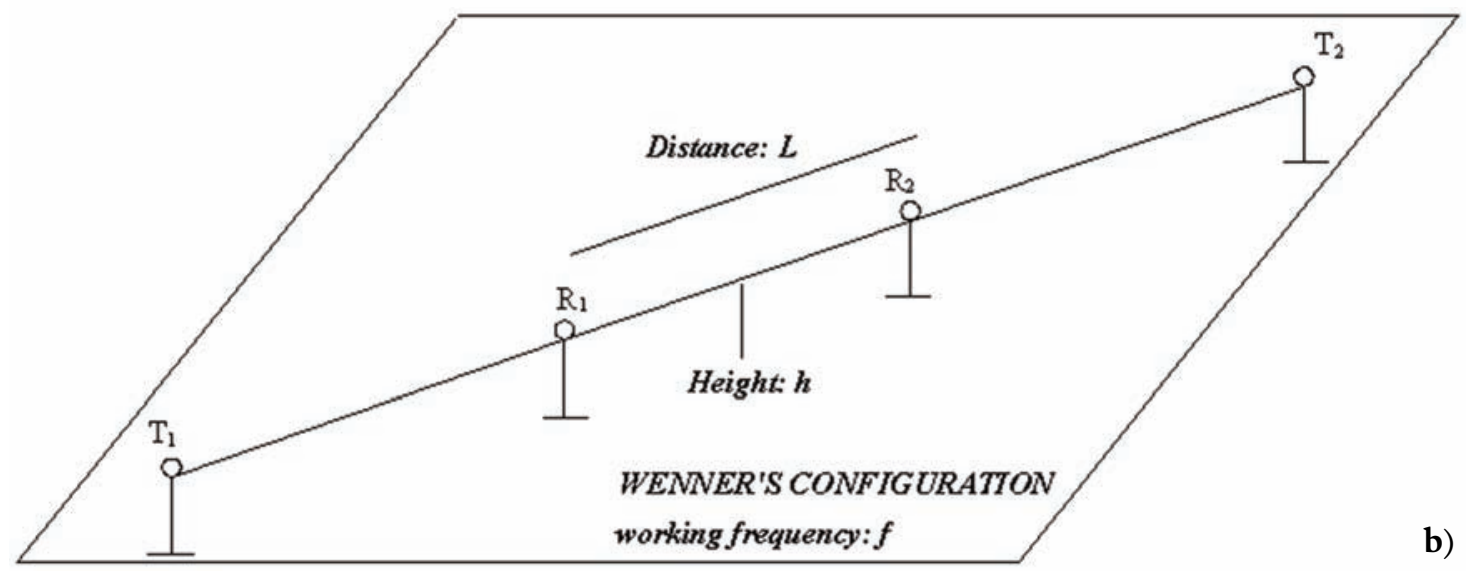

SUBSURFACE

Electrical Conductivity:

Dielectric Permittivity: $\quad \varepsilon_{r}$

Figure 6. Quadrupolar probe in square (a) and linear (Wenner's) (b) configurations.

minimum limit of inaccuracy $\Delta \varepsilon_{r} /\left.\varepsilon_{r}\right|_{\min , U}\left(\varepsilon_{r}, n\right)$. This minimum limit of inaccuracy depends approximately on both $\varepsilon_{r}$ and $n$, as it is directly proportional to the factor $\left(1+1 / \varepsilon_{r}\right)$, while decreasing as the power function $2^{-(n-1)}$ of $n$, similar to IQ sampling. The minimum value of inaccuracy $\Delta \varepsilon_{r} /\left.\varepsilon_{r}\right|_{\text {min,U }}\left(\varepsilon_{r}, n\right)$ in the uniform mode is higher than the minimum inaccuracy $\Delta \varepsilon_{r} /\left.\varepsilon_{r}\right|_{\text {min,IQ }}\left(\varepsilon_{r}, n\right)$ corresponding to the IQ mode (Figures 2a, 3; Table 1), i.e.:

$$
\begin{gathered}
\frac{\Delta \varepsilon_{r}}{\varepsilon_{r}} \geq\left.\frac{\Delta \varepsilon_{r}}{\varepsilon_{r}}\right|_{\text {min, } U} \cong\left(1+\frac{1}{\varepsilon_{r}}\right) \frac{1}{2^{n-1}}\left[1+\left(\frac{f_{T}}{f_{o p t, U}}\right)^{2}\right]>> \\
>>\left.\frac{1}{4} \frac{\Delta \varepsilon_{r}}{\varepsilon_{r}}\right|_{\text {min }, I Q}, f_{o p t, U}<<f_{T} .
\end{gathered}
$$

Finally, the minimum value of frequency $f_{U, \text { min }}\left(f_{T}, n\right)$ that allows an inaccuracy $\Delta \varepsilon_{r} / \varepsilon_{r}\left(f, f_{S}, n\right)$ below a prefixed limit $\Delta \varepsilon_{r} /\left.\varepsilon_{r}\right|_{\text {fixed }}(10 \%)$ depends both on $f_{T}\left(\sigma, \varepsilon_{r}\right)$ and $n$, as it is directly proportional to $f_{T}$, while it decreases as a power function of $n$, such that (Figure 5):

$$
f_{\text {min }, U} \cong \frac{f_{T}}{\sqrt{\frac{\Delta \varepsilon_{r} /\left.\varepsilon_{r}\right|_{\text {fixed }}}{\Delta \varepsilon_{r} /\left.\varepsilon_{r}\right|_{\text {min }, U}}-1}}<f_{\text {opt }, U} .
$$

To conclude also on the uniform mode, the optimal frequency and the band of frequency of the quadrupolar probe interact in a competitive way. Indeed, an ADC with a high bit resolution is characterized by a low sampling rate, for which having selected the subjacent medium to be analyzed, the higher the resolution of the sampler used, with the aim of shifting the optimal frequency of the quadrupolar probe into a low band, the more the low sampling rate has the self-defeating effect of narrowing its frequency band. Moreover, a material with low electrical conductivity is usually characterized by low dielectric permittivity. Having designed the ADC, the more the quadrupole measures a transfer impedance limited by a low cut-off frequency, the more it can work at a low optimal frequency, even if centered 
SOIL

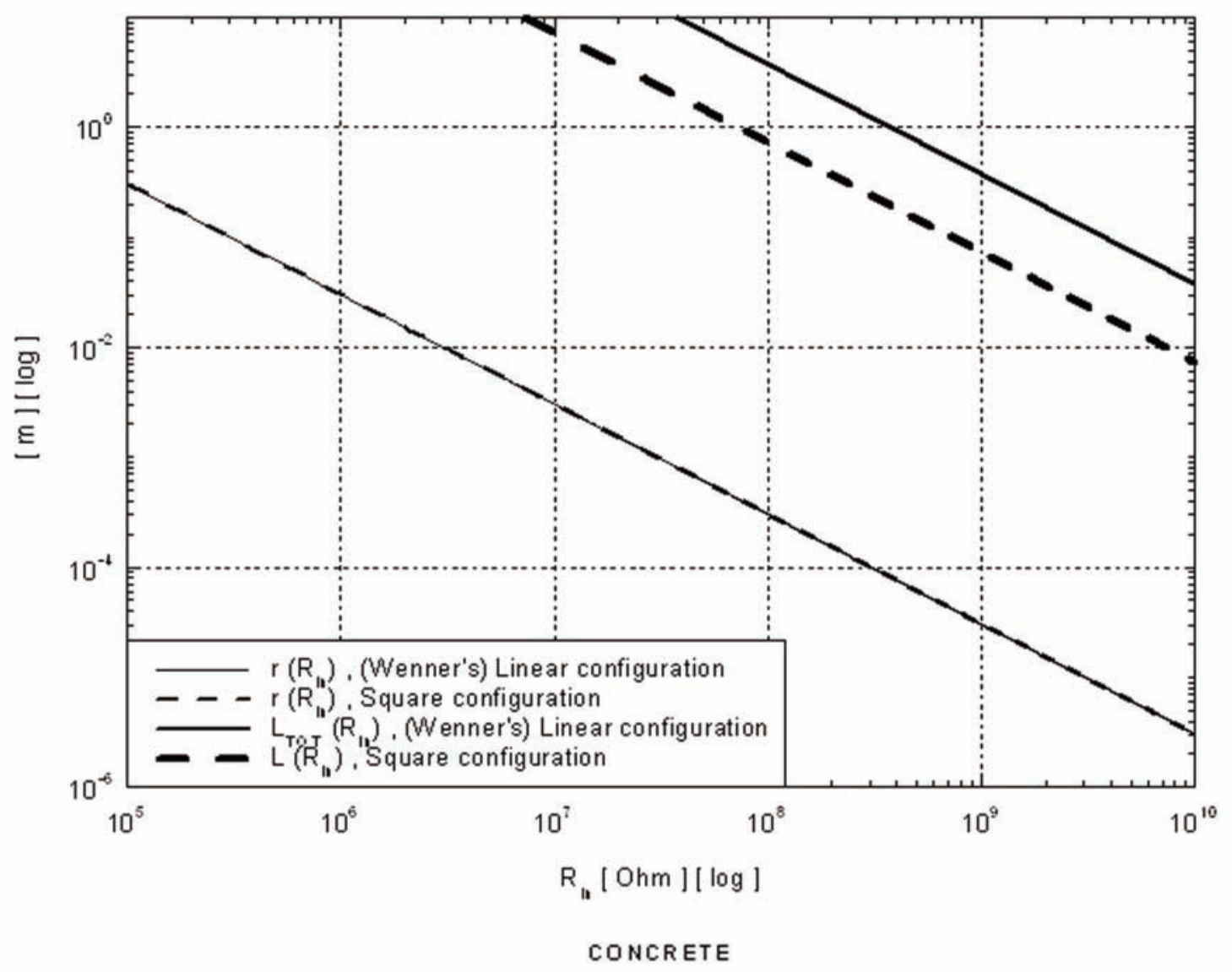

a)

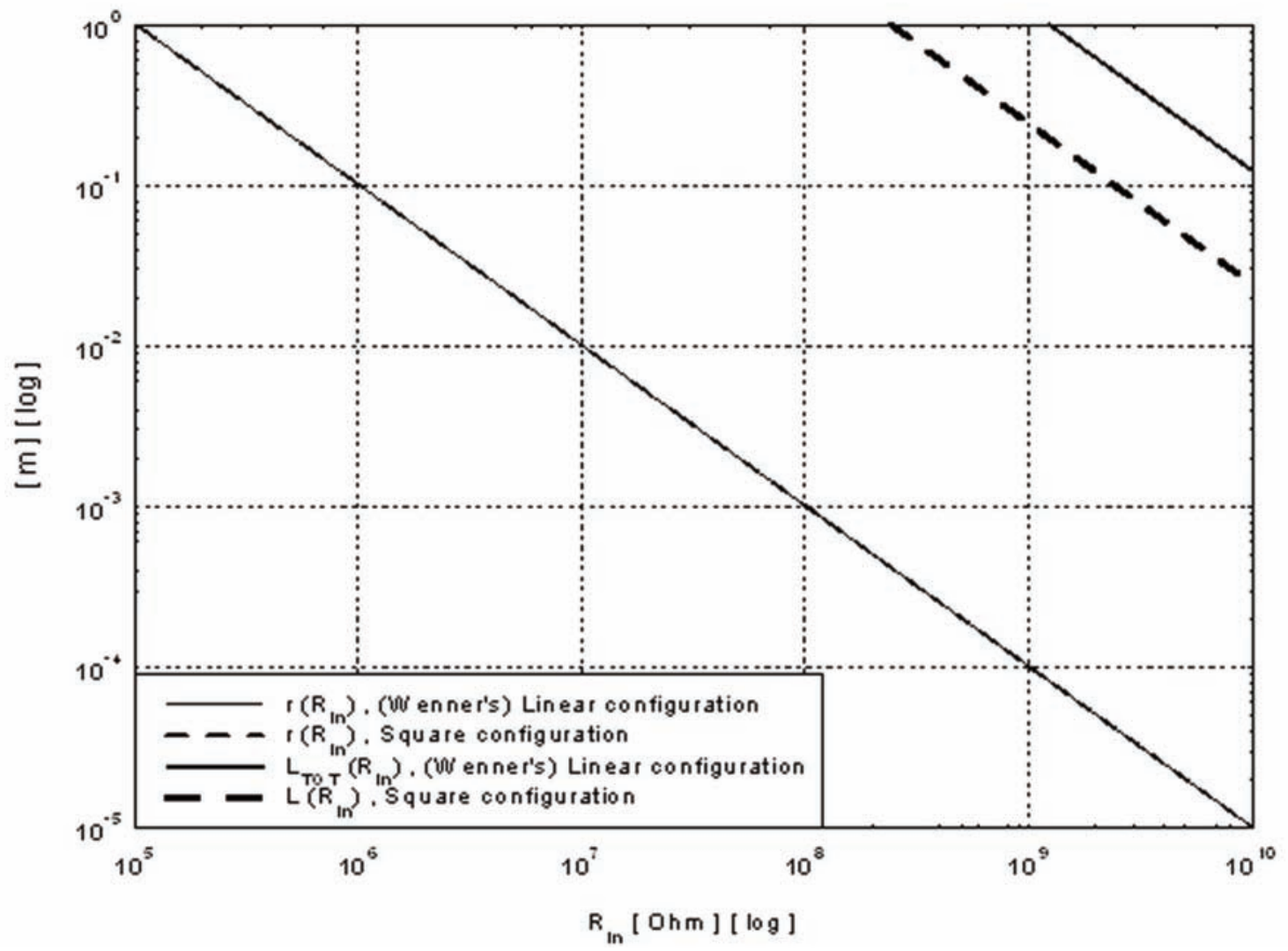

b)

Figure 7. With reference to the operating conditions described in the caption to Figure 2 (for quadrupolar probe); the quadrupole is connected to an IQ sampler of minimum bit resolution $n_{\min }=12$ that allows inaccuracies in the measurements below a prefixed limit $(10 \%)$ within the band $\left[f_{\min }, B\right]$ from the minimum value of frequency $f_{\text {min }}$ to $B=100 \mathrm{kHz}$. Like-Bode's diagrams of the radius $r\left(R_{\text {in }}, f_{\text {min }}\right)$ for probe electrodes and of the characteristic geometrical dimension $L_{s}\left(r, n_{\text {min }}\right)$ for square and of the length $L_{T O T}\left(r, n_{\text {min }}\right)=3 \cdot L_{W}\left(r, n_{\text {min }}\right)$ for (Wenner's) linear configurations, plotted as functions of the input resistance $R_{i n}$ for the amplifier stage, for nonsaturated terrestrial soil (a) and concrete (b); (Table 2 ). 


\begin{tabular}{ccc}
\hline & Square configuration & $\begin{array}{c}\text { (Wenner's) } \\
\text { Linear configuration }\end{array}$ \\
\hline Soil & & \\
$\left(\boldsymbol{\varepsilon}_{r}=4, \boldsymbol{\rho}=\mathbf{3 0 0 0} \boldsymbol{\Omega} \cdot \boldsymbol{m}\right)$ & & \\
$L, L_{\mathrm{TOT}}=3 \cdot L$ & $\mathrm{~L} \approx 1 \mathrm{~m}$ & $\mathrm{~L}_{\mathrm{TOT}} \approx 10 \mathrm{~m}$ \\
$R_{\text {in }}$ & $72.8 \mathrm{M} \Omega$ & $37.3 \mathrm{M} \Omega$ \\
$r$ & $417.042 \mu \mathrm{m}$ & $813.959 \mu \mathrm{m}$ \\
\hline
\end{tabular}

$\begin{array}{ccc}\text { Concrete } & & \\ \left(\boldsymbol{\varepsilon}_{\boldsymbol{r}}=\mathbf{4} \boldsymbol{\rho}=\mathbf{1 0 0 0 0} \boldsymbol{\Omega} \cdot \boldsymbol{m}\right) & \mathrm{L} \approx 1 \mathrm{~m} & \mathrm{~L}_{\mathrm{TOT}} \approx 1 \mathrm{~m} \\ L, L_{\mathrm{TOT}}=3 \cdot L & 242 \mathrm{M} \Omega & 1.24 \mathrm{G} \Omega \\ R_{\text {in }} & 418.197 \mu \mathrm{m} & 81.616 \mu \mathrm{m} \\ r & \end{array}$

Table 2. The radius $r$ of the probe electrodes and characteristic geometrical dimension of the quadrupole for the square (side $L$ ) or Wenner's (length $L_{\text {TOT }}=3 L$ ) configurations, using an amplifier stage with input resistance $R_{\text {in }}$ that is connected to an IQ sampling ADC with bit resolution $n=12$, to perform measurements on soil or concrete.

on a narrow band. Finally, having selected the medium to be analyzed and having designed the sampler, the more the frequency band of the probe is widened, the more the inaccuracy of the measurements is increased.

\section{Characteristic geometrical dimensions of the quadrupolar probe}

In this section, we refer to Vannaroni et al. [2004], who discussed the dependence of the transmitting (TX) current and the reading $(\mathrm{RX})$ voltage on the array and electrode dimensions. The dimensions of the quadrupolar probe terminals are not critical in the definition of the system, because they can be considered point electrodes with respect to their separating distances. In this respect, the separating distances to consider are either the square array side (Figure 6a) or the spacing distance for a Wenner configuration (Figure 6b). The only aspect that can be of importance for the practical implementation of the system is the relationship between the electrode dimensions and the magnitude of the current injected into the ground. The current is a critical parameter of the mutual impedance probe, in that in general, given the practical voltage levels applicable to the electrodes and the capacitive coupling with the soil, the current levels are expected to be quite low, with a resulting limit to the accuracy that can be achieved for the amplitude and phase measurements. Furthermore, low currents imply a reduction in the voltage signal read across the RX terminals and more stringent requirements for the reading amplifier.

The Appendix shows that once the input resistance $R_{\text {in }}$ of the amplifier stage is fixed and the minimum value of frequency $f_{\min }$ for the quadrupole is selected (Figure 1), which allow an inaccuracy in the measurement of the dielectric permittivity $\varepsilon_{r}$ below a prefixed limit (10\%), then the radius
$r\left(R_{\text {in }}, f_{\min }\right)$ of the electrodes can be designed, as this depends only on the resistance $R_{\text {in }}$ and the frequency $f_{\text {min }}$, as the radius is an inversely proportional function to both $R_{\text {in }}$ and $f_{\text {min }}$ (Figure 7; Table 2), as:

$$
r \cong \frac{1}{(2 \pi)^{2} \varepsilon_{0} R_{\text {in }} f_{\min }} .
$$

Moreover, once the minimum bit resolution $n_{\min }$ for the IQ or uniform sampling ADC is known, which allows an inaccuracy for permittivity $\varepsilon_{r}$ below the limit of $10 \%$, then the electrode-electrode distance $L\left(r, n_{\min }\right)$ can also be defined, as this depends only on the radius $r\left(R_{\text {in }}, f_{\text {min }}\right)$ and the bit resolution $n_{\min }$, with the distance directly proportional to $r\left(R_{\text {in }}, f_{\text {min }}\right)$ and increasing as the power function $2^{n_{\text {min }}}$ of $n_{\text {min }}$ (Figure 7; Table 2),

$$
L \propto r \cdot 2^{n_{\min }} .
$$

Finally, the radius $r\left(R_{\text {in }}, f_{\text {min }}\right)$ remains invariant whether the probe assumes a linear (Wenner) or a square configuration. Once the resolution nmin is also given, then the distance $L_{S}\left(r, n_{\min }\right)$ in the square configuration must be smaller by a factor $\left(2-2^{1 / 2}\right)$ compared to the corresponding distance $L_{W}\left(r, n_{\text {min }}\right)$ in the Wenner configuration [Settimi et al. 2010]:

$$
L_{S}=(2-\sqrt{2}) \cdot L_{W}=(2-\sqrt{2}) \cdot r \cdot 2^{n_{\min }} .
$$

\section{Concluding discussion}

This report has discussed the development and engineering of a suitable quadrupolar probe for simultaneous and noninvasive surveys of electrical resistivity and dielectric permittivity. The quadrupolar probe can perform measurements on a subsurface with inaccuracies below a fixed limit $(10 \%)$ in a LF bandwidth $(100 \mathrm{kHz})$. The quadrupole should be connected to an appropriate ADC that can sample 
in IQ or in uniform mode. If the probe is characterized by galvanic contact with the surface, the inaccuracies in the measurements of resistivity and permittivity due to the IQ or uniform sampling ADC have been expressed analytically. A large number of numerical simulations have shown that the performance of the probe depends on the selected sampler, and that the IQ is better compared to the uniform mode under the same operating conditions, i.e. for bit resolution and medium (Figure 3; Table 1).

For the IQ mode, this is specified by an inaccuracy $\Delta \Phi_{Z} / \Phi_{Z}\left(n_{\text {IQ }}\right)$ for the phase of the transfer impedance, which depends only on the bit resolution $n_{\mathrm{IO}}$, assuming small values (e.g., $\Delta \Phi_{Z} /\left.\Phi_{Z}\right|_{\max }(12)=1 / 2^{12} \approx 2.4 \cdot 10^{-4}$ ] over the entire frequency band $B=100 \mathrm{kHz}$ of the quadrupole. For the uniform mode, the corresponding inaccuracy $\Delta \Phi_{Z} / \Phi_{Z}\left(f, f_{S}\right)$ depends on the frequency $f$, and it is only small for frequencies much lower than the sampling rate $f_{S}$ [i.e. $2 f / f_{S} \leq$ $\left.\Delta \Phi_{Z} /\left.\Phi_{Z}\right|_{\max }(12)\right]$. The principal advantages of the IQ mode are: first, the minimum value $f_{\min }(n)$ of the frequency, which allows an inaccuracy for permittivity $\varepsilon_{r}$ below $10 \%$, is slightly lower when the probe is connected to an IQ rather than a uniform $\mathrm{ADC}$, if all other operating conditions are equal, i.e. the resolution and the surface; and secondly, the inaccuracy $\Delta \sigma / \sigma$ for conductivity $\sigma$ calculated in $f_{\text {min }}(n)$ is smaller using IQ than with uniform sampling, as $\Delta \sigma /\left.\sigma\right|_{I Q}<\Delta \sigma /\left.\sigma\right|_{\mathrm{U}}$, by almost one order of magnitude under the same operating conditions, and in particular for the resolution. As a minor disadvantage, the optimal frequency $f_{\text {opt }}$, which minimizes the inaccuracy for $\varepsilon_{r}$, is generally higher using IQ than uniform sampling, as $f_{o p t, I Q}>f_{o p t, U}$ of almost one MF decade, at most, under the same operating conditions, and in particular for the surface (Figure 3).

Instead, the uniform mode is specified by two degrees of freedom, the resolution of bit $n_{U}$ and the rate of sampling $f_{S}$, compared to the IQ mode, which is characterized by one degree of freedom, the bit resolution $n_{\mathrm{IO}}$. Consequently the quadrupolar probe can be connected to a uniform ADC with a sampling rate $f_{S}$ sufficiently fast at a resolution (for example $n_{U}=8$ ) lower than for the IQ (i.e. $n_{\mathrm{IQ}}=12$ ) that it reaches the same prefixed limit of inaccuracy (i.e. $10 \%$ ) in the measurement of the dielectric permittivity $\varepsilon_{r}$, for various media, and especially for those media with low electrical conductivities $\sigma$ (Table 1).

\section{References}

Arpaia, P., P. Daponte and L. Michaeli (1999). Influence of the architecture on ADC error modelling, IEEE T. Instrum. Meas., 48 (5), 956-966.

Arpaia, P., P. Daponte and S. Rapuano (2004). A state of the art on ADC modelling, Comput. Stand. Int., 26 (1), 31-42.

Declerk, P. (1995). Bibliographic study of georadar principles, applications, advantages, and inconvenience, NDT \& E Int., 28, 390-442 (French, with English abstract).
Del Vento, D., and G. Vannaroni (2005). Evaluation of a mutual impedance probe to search for water ice in the Martian shallow subsoil, Rev. Sci. Instrum., 76 (8), 084504084504-8.

Grard, R. (1990a). A quadrupolar array for measuring the complex permittivity of the ground: application to earth prospection and planetary exploration, Meas. Sci. Technol., 1 (3), 295-301.

Grard, R. (1990b). A quadrupole system for measuring in situ the complex permittivity of materials: application to penetrators and landers for planetary exploration, Meas. Sci. Technol., 1 (8), 801-806.

Grard, R., and A. Tabbagh (1991). A mobile four electrode array and its application to the electrical survey of planetary grounds at shallow depth, J. Geophys. Res., 96 (B3), 4117-4123.

Jankovic, D., and J. Öhman (2001). Extraction of In-phase and Quadrature Components by IF-sampling, Master Thesis, Department of Signals and Systems, Cahlmers University of Technology, Goteborg (carried out at Ericson Microwave System AB), 80 pp.

Mojid, M.A., G.C.L. Wyseure and D.A. Rose (2003). Electrical conductivity problems associated with time-domain reflectometry (TDR) measurement in geotechnical engineering, Geotech. Geo. Eng., 21 (3), 243-258.

Mojid, M.A., and H. Cho (2004). Evaluation of the time-domain reflectometry (TDR)-measured composite dielectric constant of root-mixed soils for estimating soil-water content and root density, J. Hydrol., 295 (1-4), 263-275.

Polge, R.J., B.K. Bhagavan and L. Callas (1975). Evaluating analog-to-digital converters, Simulation, 24 (3), 81-86.

Razavi, B. (1995). Principles of Data Conversion System Design, Wiley-IEEE Press, New York, 272 pp.

Samouëlian, A., I. Cousin, A. Tabbagh, A. Bruand and G. Richard (2005). Electrical resistivity survey in soil science: a review, Soil Till. Res., 83 (2), 172-193.

Sbartaï, Z. M., S. Laurens, J. P. Balayssac, G. Arliguie and G. Ballivy (2006). Ability of the direct wave of radar groundcoupled antenna for NDT of concrete structures, NDT \& E Int., 39 (5), 400-407.

Settimi, A., A. Zirizzotti, J.A. Baskaradas and C. Bianchi (2009). Optimal requirements of a data acquisition system for a quadrupolar probe employed in electrical spectroscopy, Earth-prints.org: http:/ / hdl.handle.net/2122/5176; arXiv. org: arXiv:0908.0648 [physics.geo-ph].

Settimi, A., A. Zirizzotti, J.A. Baskaradas and C. Bianchi (2010). Inaccuracy assessment for simultaneous measurements of resistivity and permittivity applying sensitivity and transfer function approaches, Annals of Geophysics, 53 (2), 119 (pre-publication paper available since 2009 on Earth-prints.org: http://hdl.handle.net/2122/5180; and on arXiv.org: arXiv:0908.0641 [physics.geo-ph]).

Tabbagh, A., A. Hesse and R. Grard (1993). Determination 
of electrical properties of the ground at shallow depth with an electrostatic quadrupole: field trials on archaeological sites, Geophys. Prospect., 41 (5), 579-597.

Vannaroni, G., E. Pettinelli, C. Ottonello, A. Cereti, G. Della Monica, D. Del Vento, A. M. Di Lellis, R. Di Maio, R. Filippini, A. Galli, A. Menghini, R. Orosei, S. Orsini, S. Pagnan, F. Paolucci, A. Pisani R., G. Schettini, M. Storini and G. Tacconi (2004). MUSES: multi-sensor soil electromagnetic sounding, Planet. Space Sci., 52 (1-3), 67-78.

*Corresponding author: Alessandro Settimi,

Istituto Nazionale di Geofisica e Vulcanologia, Sezione Roma 2,

Roma, Italy; e-mail: alessandro.settimi@ingv.it.

(C) 2010 by the Istituto Nazionale di Geofisica e Vulcanologia. All rights reserved. 


\section{Appendix}

A series of two spherical capacitors with radius $r$ and spacing distance $L>>r$ is characterized by the electrical capacitance:

$$
C=\frac{2 \pi \varepsilon_{0}}{\frac{1}{r}-\frac{1}{L-r}} \cong 2 \pi \varepsilon_{0} r, \text { for } L>>r,
$$

with $\varepsilon_{0}$ the dielectric constant in a vacuum.

A quadrupolar probe with four spherical electrodes of radius $r$ and separating distance $L>>r$ is arranged in the Wenner configuration with total length $L_{t o t}=3 L$, which is specified by the pairs of transmitting electrodes $T_{1}$ and $T_{2}$ at the ends of the quadrupole and the reading electrodes $R_{1}$ and $R_{2}$ in the middle of the probe. The quadrupolar probe is characterized by a capacitance that is almost invariant for the pairs of electrodes $T_{1,2}$ and $R_{1,2}$,

$$
\begin{aligned}
C_{T_{1}, T_{2}}= & \frac{2 \pi \varepsilon_{0}}{\frac{1}{r}-\frac{1}{3 L-r}} \cong C_{R_{1}, R_{2}}=\frac{2 \pi \varepsilon_{0}}{\frac{1}{r}-\frac{1}{L-r}} \cong \\
& \cong C=2 \pi \varepsilon_{0} r \quad \text { for } L>>r .
\end{aligned}
$$

As the charge $Q$ of the electrodes are equal, the electrical voltage across the pair $T_{1}$ and $T_{2}$ approximates the voltage between $R_{1}$ and $R_{2}$,

$$
\Delta V_{T_{1}, T_{2}} \cong \Delta V_{R_{1}, R_{2}} \cong \frac{Q}{C} .
$$

For the equivalent capacitance circuit that schematizes the transmission stage of the quadrupole (Figure 8a), if the effect of the capacitance $C$ across the electrodes is predominant relative to the shunted capacitances $C_{T 1}$ and $C_{T 2}$, describing the electrical coupling between the transmitting electrodes and the subjacent medium as,

$$
C<<C_{T_{1}}=C_{T_{2}},
$$

then, working in the frequency $f$, the probe injects into the medium a minimum bound for the modulus of the current $|I|_{\text {min }}$,

$$
\begin{gathered}
|I|_{\text {min }} \cong \omega \cdot C \cdot \Delta V_{T_{1}, T_{2}}=2 \pi f \cdot 2 \pi \varepsilon_{0} r \cdot \Delta V_{T_{1}, T_{2}}= \\
=(2 \pi)^{2} \varepsilon_{0} r f \Delta V_{T_{1}, T_{2}},
\end{gathered}
$$

with $|I|_{\min }$ increasing linearly with $f$.

For the equivalent capacitance circuit that represents the reception stage of the quadrupolar probe (Figure $8 b$ ), the effect of the capacitance $C$ across the electrodes is predominant even relative to the shunted capacitances $C_{R 1}$ and $C_{R 2}$, describing the coupling between the reading electrodes and the subjacent medium as,

$$
C<<C_{R_{1}}=C_{R_{2}} \text {, }
$$

If the quadrupole of electrode-electrode distance $L$ is
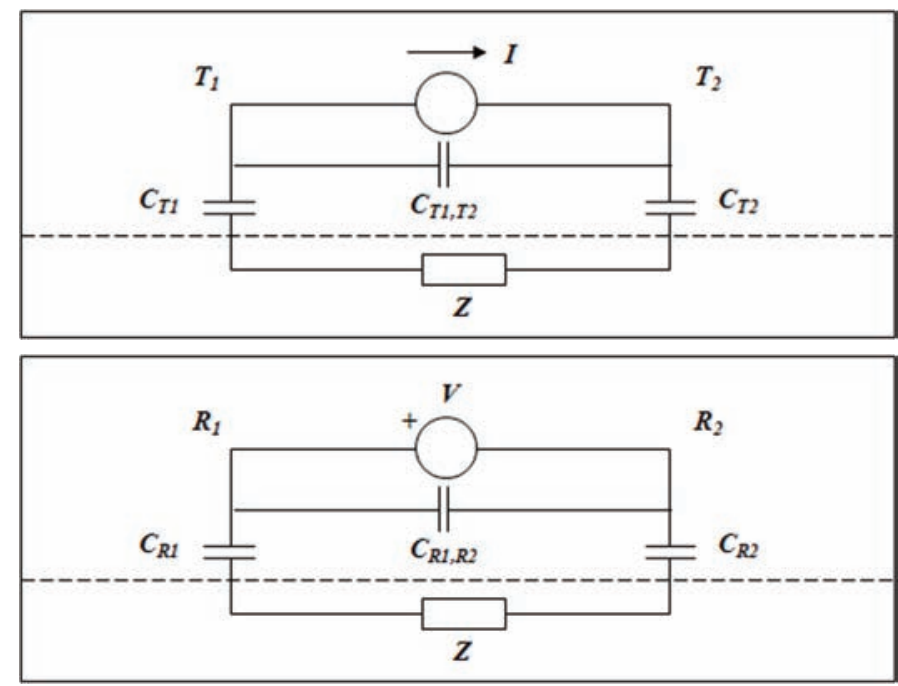

Figure 8. Equivalent capacitance circuits of the quadrupolar probe that schematize the transmission (a) and reception (b) stages.

immersed in vacuum, then it can be characterized by the vacuum capacitance:

$$
C_{0}=4 \pi \varepsilon_{0} L
$$

and, in the frequency $f$, measures a minimum limit $|Z|_{\text {min }}$ for the transfer impedance in modulus,

$$
|Z|_{\min }=\frac{1}{\omega \cdot C_{0}}=\frac{1}{2 \pi f \cdot 4 \pi \varepsilon_{0} L}=\frac{1}{2(2 \pi)^{2} \varepsilon_{0} L f},
$$

which gives rises to a minimum for the electrical voltage $\Delta V_{\text {min, R1,R2 }}$, flowing the current $|I|_{\text {min }}$,

$$
\begin{aligned}
& \Delta V_{R_{1}, R_{2}}^{\min }=|Z|_{\min }|I|_{\min } \cong \\
& \cong \frac{1}{2(2 \pi)^{2} \varepsilon_{0} L f} \cdot(2 \pi)^{2} \varepsilon_{0} L f \Delta V_{T_{1}, T_{2}}=\frac{1}{2} \frac{r}{L} \Delta V_{T_{1}, T_{2}} .
\end{aligned}
$$

Note that $\Delta V_{\text {min, R1,R2 }}$ is independent of $f$, as $|I|_{\text {min }}$ is directly and $|Z|_{\min }$ inversely proportional to $f$.

As a first finding, downstream of the probe the ADC must be specified by a bit resolution $n$, such that:

$$
\frac{\Delta V_{R_{1}, R_{2}}^{\min }}{\Delta V_{T_{1}, T_{2}}} \cong \frac{1}{2} \frac{r}{L} \approx \frac{\Delta V_{R_{1}, R_{2}}^{\min }}{\Delta V_{R_{1}, R_{2}}}=\frac{1}{2^{n+1}} \Rightarrow \frac{r}{L} \approx \frac{1}{2^{n}} .
$$

Instead, if the quadrupole shows galvanic contact with a medium of electrical conductivity $\sigma$ and dielectric permittivity $\varepsilon_{r}$ working in a band lower than the cut-off frequency, i.e. $f_{T}=f_{T}\left(\sigma, \varepsilon_{r}\right)=1 / 2 \pi \cdot \sigma / \varepsilon_{0}\left(\varepsilon_{r}+1\right)$, then it measures the transfer impedance in the modulus

$$
|Z|=\frac{1}{2 \pi \sigma L} .
$$

As final findings, the voltage amplifier downstream of the probe must be specified by an input resistance $R_{i n}$ that is larger than both the transfer impedance, i.e.

$$
R_{\text {in }}>|Z|=\frac{1}{2 \pi \sigma L} \Rightarrow L>\frac{1}{2 \pi \sigma R_{\text {in }}},
$$


and the reactance associated with the capacitance $C$, which is characterized by a maximum value in the minimum of frequency $f_{\text {min }}$, i.e.

$$
\begin{aligned}
& R_{\text {in }}>\frac{1}{\omega_{\text {min }} C}=\frac{1}{2 \pi f_{\min } \cdot 2 \pi \varepsilon_{0} r}= \\
& =\frac{1}{(2 \pi)^{2} \varepsilon_{0} r f_{\min }} \Rightarrow r>\frac{1}{(2 \pi)^{2} \varepsilon_{0} R_{i n} f_{\min }} .
\end{aligned}
$$

